\title{
EXISTENCE AND UNIQUENESS RESULT FOR THE PROBLEM OF VISCOUS FLOW IN A GRANULAR MATERIAL WITH A VOID
}

\author{
BY \\ MIRELA KOHR (Faculty of Mathematics and Computer Science, Babeş-Bolyai University, 1 M. \\ Kogălniceanu Str., 400084 Cluj-Napoca, Romania) \\ AND \\ G. P. RAJA SEKHAR (Department of Mathematics, Indian Institute of Technology Kharagpur, \\ Kharagpur 721 302, India)
}

\begin{abstract}
The purpose of this paper is to obtain an indirect boundary integral formulation for the three-dimensional viscous flow problem in a granular material with a void. The corresponding existence and uniqueness result of the classical solution to this problem is proved by using the theory of hydrodynamic potentials.
\end{abstract}

1. Introduction. Due to its increasing applications in chemical engineering, oil reservoirs, and geology, the study of incompressible viscous flow in a porous medium has gained a lot of attention. Fluid flow at the interfacial region in systems that consist of a fluid saturated porous medium and an adjacent fluid layer occurs in a wide variety of physical and engineering applications. Generally, there are three interface zones the interface region between two porous media, the interface region between a porous medium and a fluid, and the interface region between a porous medium and an impermeable medium. Few examples are where the oil flow in petroleum reservoirs encounters different layers of sand, rock, shale, lime stone, etc. Similar situations can be observed in geothermal operations, thermal insulation in buildings, etc. There are extensive studies on viscous flow past porous regions. The problem of Stokes flow past porous particles using the Brinkman equation began with the work of Higdon and Kojima [8]. Also Qin and Kaloni [24] have discussed the creeping flow past a porous spherical shell. For axisymmetric flows, there were studies where the corresponding stream function for Stokes equations was used ([5], [37], [38]). In the case of arbitrary viscous flow past various porous spherical objects, Palanippan [26], Padmavathi et al. [25], and Raja Sekhar et.

Received April 17, 2006.

2000 Mathematics Subject Classification. Primary 76D; Secondary 76M.

Key words and phrases. Stokes equation, Brinkman equation, boundary value problems, fundamental solution, potential theory, boundary integral representations, existence and uniqueness result.

E-mail address: mkohr@math.ubbcluj.ro

E-mail address: rajas@maths.iitkgp.ernet.in 
al. [31], [32], [36] have contributed using the corresponding complete general solutions of Stokes equations and Brinkman equations.

The study of viscous flow past porous structures of complex geometries is very limited. Though there are various computational methods like finite differences and finite element methods which are domain discretization methods, integral equation techniques are attractive to begin the numerical solution of a wide variety of problems concerning the slow motion of viscous fluids. The aim of this paper is to give a boundary integral formulation for the problem of a viscous flow through porous media. The current problem of our interest in studying ground water, geophysical phenomena in soil mechanics and civil engineering where solid structure is maintained by arching and friction between the grains. In geological applications the collapse of a microstructure due to viscous stress causes some non-linear effects like inhomogeneity in the matrix, destruction of the cavity boundary, growth of a cavity, etc. Sano [30] and Raja Sekhar and Sano [34] have calculated the flow around a two-dimensional (2D) hole which has a circular boundary and a slightly but symmetrically deformed one respectively, which shows that the effect of the hole increases with the value of $\zeta_{0}=R_{0} / \sqrt{k}$, where $R_{0}$ is the characteristic void radius and $k$ is the permeability of the porous medium. At the same time, as an application to measure groundwater velocity, they made a theoretical study on the viscous flow past a spherical void in porous media which is the corresponding three dimensional (3D) problem [33]. Because in practical applications a bore hole might not be a complete circle or sphere, to answer the question on whether the velocity at the center or volume flux into the cavity is sensitive to the deformation of the cavity boundary, Raja Sekhar and Sano [35] studied the viscous flow in a granular material with a void of arbitrary shape in the $2 \mathrm{D}$ case. They have expanded the boundary to represent the deformation of the cavity and calculated the flow fields both inside and outside the cavity up to the second order of the deformation parameter. In [33] and [35] the Stokes system is used for the flow inside the cavity and the Brinkman equation for the flow in the porous region, and the flow field is computed by matching the continuity of velocity and stress (see also $[1,9,21,22,23])$

The theory of boundary integral equations offers attractive methods as a basis for the study of a wide variety of problems concerning viscous incompressible flows at low Reynolds numbers past or due to the motion of solid particles or fluid interfaces (see e.g. [11], [15]). Green's representation formula analogous to those employed in the potential theory is well known for Stokes flow (see e.g. [15], Chapter 3). This formula has been extensively used in the numerical solution of various Stokes flow problems since the original work of Youngren and Acrivos [43]. The application of this formula to the case of exterior Stokes problems of Dirichlet type (for which the boundary velocity is prescribed) leads to a Fredholm integral equation of the first kind for the unknown boundary traction. However, it is known that the Fredholm integral equations of the first kind generally give rise to unstable numerical schemes based upon the discretization of the surface involved. On the other hand, the numerical solution corresponding to a well-posed Fredholm integral equation of the second kind is always stable. For a threedimensional exterior Stokes problem with a prescribed boundary velocity (i.e., an exterior boundary value problem of the Dirichlet type for the Stokes system), Power and Miranda 
[28] remarked that the integral representation of the velocity field in terms of a doublelayer potential without any additional term can represent only those Stokes flows which exert zero total force and torque on the boundary of the flow domain. This representation may be completed by adding some terms in a linear combination that lead to arbitrary total force and torque. Karrila and Kim [10] called the method of Power and Miranda the Completed Double Layer Boundary Integral Equation Method (CDLBIEM), in view of the fact that it involves the idea of completing the double-layer integral operator. Botte and Power [2] obtained an extension of Power and Miranda's indirect method for threedimensional interior Stokes flow problems. Also Power [27] extended the CDLBIEM from the case of three-dimensional exterior Stokes flow problems to the case of two-dimensional exterior Stokes flow problems. Briceno and Power [3] obtained a completed boundary integral equation approach for the numerical solution of the boundary value problem corresponding to the motion of $N$ solid particles in the interior of a deformable viscous drop. Kohr [12] obtained a boundary integral method in order to study the oscillatory Stokes flow due to the oscillations of two solid spheres in an incompressible Newtonian fluid. On the other hand, Varnhorn [39], [40], [41] developed a complete potential theory for the Stokes resolvent system whose unknowns are defined only on domains in $\mathbb{R}^{n}$ with connected boundaries. The method developed by Kohr [12] leads to the idea of the extension of the potential theory for the Stokes resolvent system from the case of domains in $\mathbb{R}^{n}$ with connected boundaries to the general case of domains in $\mathbb{R}^{n}(n \geq 2)$ with non-connected boundaries (see [13], [14]). Recently, Kohr [13] used this extension in order to obtain an indirect boundary integral method for a mixed boundary value problem associated to the (unsteady) Stokes resolvent system in a bounded domain in $\mathbb{R}^{n}$ with compact but non-connected boundary. The corresponding steady case has been treated by Briceno and Power [3]. More recently, Kohr [14] obtained the existence and uniqueness results for the Dirichlet problems associated to the Stokes resolvent system in bounded and exterior domains in $\mathbb{R}^{n}$ with compact but non-connected boundaries by using some indirect boundary integral representations. In addition, the corresponding boundary integral methods extend the CDLBIEM developed by Power and Miranda [28] for unbounded Stokes flow problems to the case of unsteady Stokes flow problems in bounded and exterior domains.

Since the Brinkman equation, which describes the flow in porous media, is mathematically equivalent to the (unsteady) Stokes resolvent equation, the potential theory developed for the Stokes resolvent system may be applied to the system of continuity and Brinkman equations in order to study various flows in porous media.

The purpose of this paper is to obtain an indirect boundary integral formulation for the three-dimensional viscous flow problem in a granular material with a void. The flow in the granular material is described by the continuity and Brinkman equations, and the flow inside the void is governed by the Stokes system of equations. We use indirect boundary integral representations that reduce the physical problem to a system of Fredholm integral equations of the second kind with a unique continuous solution.

2. The mathematical formulation of the problem. Let us consider an otherwise unbounded homogeneous granular material in which a void occupying the bounded 
domain $D_{0} \subset \mathbb{R}^{3}$ is given. Let us denote by $D_{e}$ the unbounded domain, exterior to the boundary $\Gamma$ of $D_{0}$, and assume that the fluid flow far from the void is a uniform one with velocity and pressure fields $\mathbf{U}_{\infty}$ and $p_{\infty}$ respectively (see Fig. 1). Assume that the boundary $\Gamma$ of the void is slightly deformed such that at each moment in time it remains a closed Lyapunov surface of class $C^{(2, \alpha)}$, where $\alpha \in(0,1]$.

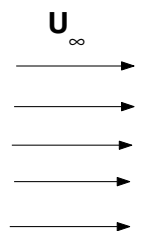

$$
D_{e}
$$

FIG. 1. Viscous flow past a void in a granular material.

Next, we assume that the flow in the granular material (i.e., in the exterior domain $D_{e}$ ) is governed by the generalized Darcy equation (or the Brinkman equation), i.e., the volume averaged velocity field $\tilde{\mathbf{v}}^{e}$ and the pressure field $\tilde{p}^{e}$ satisfy in the porous region the following equations:

$$
\begin{gathered}
-\tilde{\nabla} \tilde{p}^{e}+\mu_{e f f} \tilde{\nabla}^{2} \tilde{\mathbf{v}}^{e}-\frac{\mu_{f}}{\kappa} \tilde{\mathbf{v}}^{e}=\mathbf{0} \text { in } D_{e}, \\
\tilde{\nabla} \cdot \tilde{\mathbf{v}}^{e}=0 \text { in } D_{e}
\end{gathered}
$$

where $\kappa$ is the permeability of the porous medium, $\mu_{f}$ is the viscosity coefficient of the fluid, $\mu_{e f f}$ is the effective viscosity, and $\tilde{\nabla}^{2}$ is the Laplace operator in $\mathbb{R}^{3}$, i.e.,

$$
\tilde{\nabla}^{2}=\frac{\partial^{2}}{\partial \tilde{x}_{1}^{2}}+\frac{\partial^{2}}{\partial \tilde{x}_{2}^{2}}+\frac{\partial^{2}}{\partial \tilde{x}_{3}^{2}}
$$

The flow inside the void region (i.e., in the bounded domain $D_{0}$ ) is governed by the Stokes system of equations:

$$
\begin{gathered}
-\tilde{\nabla} \tilde{p}^{i}+\mu_{f} \tilde{\nabla}^{2} \tilde{\mathbf{v}}^{i}=\mathbf{0} \text { in } D_{0}, \\
\tilde{\nabla} \cdot \tilde{\mathbf{v}}^{i}=0 \text { in } D_{0} .
\end{gathered}
$$

Note that the superscripts $e$ and $i$ refer to the external flow and respectively to the internal flow. Also we have assumed that both media, inside and respectively outside $\Gamma$, are incompressible (see the equations (2.2) and (2.4)).

Let us introduce the non-dimensional quantities

$$
\mathbf{x}=\frac{\tilde{\mathbf{x}}}{R_{0}}, \mathbf{v}^{e}=\frac{\tilde{\mathbf{v}}^{e}}{U_{\infty}}, p^{e}=\frac{\tilde{p}^{e}}{\mu_{f} U_{\infty} / R_{0}} \frac{\mu_{f}}{\mu_{e f f}}, \mathbf{v}^{i}=\frac{\tilde{\mathbf{v}}^{i}}{U_{\infty}}, p^{i}=\frac{\tilde{p}^{i}}{\mu_{f} U_{\infty} / R_{0}},
$$

where $R_{0}$ is the characteristic void radius. The non-dimensional undisturbed velocity and pressure fields are denoted by $\mathbf{U}^{\infty}$ and $p^{\infty}$. Then the governing equations (2.1)-(2.4) take 
the following non-dimensional form:

$$
\begin{gathered}
-\nabla p^{e}+\left(\nabla^{2}-\chi^{2}\right) \mathbf{v}^{e}=\mathbf{0} \text { in } D_{e} \\
\nabla \cdot \mathbf{v}^{e}=0 \text { in } D_{e} \\
-\nabla p^{i}+\nabla^{2} \mathbf{v}^{i}=\mathbf{0} \text { in } D_{0} \\
\nabla \cdot \mathbf{v}^{i}=0 \text { in } D_{0}
\end{gathered}
$$

where $\chi$ is the positive constant given by

$$
\chi=\frac{R_{0}}{\sqrt{\kappa}} \sqrt{\frac{\mu_{f}}{\mu_{e f f}}} .
$$

In the case of equal viscosities, $\mu_{f}=\mu_{e f f}$, the coefficient $\chi$ takes the form $\chi=R_{0} / \sqrt{\kappa}$.

On the boundary $\Gamma$ we employ the following boundary conditions:

$$
\begin{aligned}
& \mathbf{v}^{i}=\mathbf{v}^{e} \text { on } \Gamma, \\
& \mathbf{t}^{i}=\mathbf{t}^{e} \text { on } \Gamma,
\end{aligned}
$$

i.e., the continuity of the velocity and boundary traction fields on $\Gamma$. Note that $\mathbf{t}^{e}$ is the boundary traction corresponding to the external fields $\mathbf{v}^{e}$ and $p^{e}$, and $\mathbf{t}^{i}$ is the boundary traction due to the internal fields $\mathbf{v}^{i}$ and $p^{i}$.

Assume that the fields $\mathbf{v}^{e}-\mathbf{U}^{\infty}$ and $p^{e}-p^{\infty}$ vanish at infinity such that

$$
\begin{aligned}
& \left(\left|\mathbf{v}^{e}-\mathbf{U}^{\infty} \| \nabla\left(\mathbf{v}^{e}-\mathbf{U}^{\infty}\right)\right|\right)(\mathbf{x})=o\left(|\mathbf{x}|^{-2}\right) \text { as }|\mathbf{x}| \rightarrow \infty \\
& \left(\left|\mathbf{v}^{e}-\mathbf{U}^{\infty} \| p^{e}-p^{\infty}\right|\right)(\mathbf{x})=o\left(|\mathbf{x}|^{-2}\right) \text { as }|\mathbf{x}| \rightarrow \infty
\end{aligned}
$$

Therefore, we have obtained the boundary value problem (2.6)-(2.12) with the unknown fields $\mathbf{v}^{e}, p^{e}, \mathbf{v}^{i}$ and $p^{i}$. The purpose of this paper is to obtain a boundary integral equation method in order to show that this boundary value problem has a unique classical solution.

3. The uniqueness result of the solution. The following theorem refers to the uniqueness result of the classical solution to the boundary value problem (2.6)-(2.12).

TheOREM 3.1. The boundary value problem (2.6)-(2.12) has at most one classical solution $\left(\left(\mathbf{v}^{e}, p^{e}\right),\left(\mathbf{v}^{i}, p^{i}\right)\right) \in\left(\left(C^{2}\left(D_{e}\right) \cap C^{1}\left(\bar{D}_{e}\right)\right) \times C^{1}\left(\bar{D}_{e}\right)\right) \times\left(\left(C^{2}\left(D_{0}\right) \cap C^{1}\left(\bar{D}_{0}\right)\right) \times C^{1}\left(\bar{D}_{0}\right)\right)$.

Proof. Let us assume that the boundary value problem (2.6)-(2.12) has two classical solutions

$$
\begin{aligned}
& \left(\left(\mathbf{v}_{1}^{e}, p_{1}^{e}\right),\left(\mathbf{v}_{1}^{i}, p_{1}^{i}\right)\right) \in\left(\left(C^{2}\left(D_{e}\right) \cap C^{1}\left(\bar{D}_{e}\right)\right) \times C^{1}\left(\bar{D}_{e}\right)\right) \times\left(\left(C^{2}\left(D_{0}\right) \cap C^{1}\left(\bar{D}_{0}\right)\right) \times C^{1}\left(\bar{D}_{0}\right)\right), \\
& \left(\left(\mathbf{v}_{2}^{e}, p_{2}^{e}\right),\left(\mathbf{v}_{2}^{i}, p_{2}^{i}\right)\right) \in\left(\left(C^{2}\left(D_{e}\right) \cap C^{1}\left(\bar{D}_{e}\right)\right) \times C^{1}\left(\bar{D}_{e}\right)\right) \times\left(\left(C^{2}\left(D_{0}\right) \cap C^{1}\left(\bar{D}_{0}\right)\right) \times C^{1}\left(\bar{D}_{0}\right)\right)
\end{aligned}
$$


and let $\left(\left(\mathbf{v}_{0}^{e}, p_{0}^{e}\right),\left(\mathbf{v}_{0}^{i}, p_{0}^{i}\right)\right)$ be the difference of them. Then we have

$$
\begin{gathered}
-\nabla p_{0}^{e}+\left(\nabla^{2}-\chi^{2}\right) \mathbf{v}_{0}^{e}=\mathbf{0}, \quad \nabla \cdot \mathbf{v}_{0}^{e}=0 \text { in } D_{e} \\
-\nabla p_{0}^{i}+\nabla^{2} \mathbf{v}_{0}^{i}=\mathbf{0}, \quad \nabla \cdot \mathbf{v}_{0}^{i}=0 \text { in } D_{0}, \\
\mathbf{v}_{0}^{i}=\mathbf{v}_{0}^{e} \text { on } \Gamma \\
\mathbf{t}_{0}^{i}=\mathbf{t}_{0}^{e} \text { on } \Gamma, \\
\left(\left|\mathbf{v}_{0}^{e}\right|\left|\nabla \mathbf{v}_{0}^{e}\right|\right)(\mathbf{x})=o\left(|\mathbf{x}|^{-2}\right), \quad\left(\left|\mathbf{v}_{0}^{e}\right|\left|p_{0}^{e}\right|\right)(\mathbf{x})=o\left(|\mathbf{x}|^{-2}\right) \text { as }|\mathbf{x}| \rightarrow \infty .
\end{gathered}
$$

In view of the equations (3.1) it follows that the fields $\mathbf{v}_{0}^{e}$ and $p_{0}^{e}$ satisfy the identity (see [15], pp. 24-25)

$$
\int_{D_{e}}\left(\chi^{2}\left|\mathbf{v}_{0}^{e}\right|^{2}+2 E_{k j}\left(\mathbf{v}_{0}^{e}\right) E_{k j}\left(\mathbf{v}_{0}^{e}\right)\right) d \mathbf{x}=-\int_{\Gamma} \mathbf{v}_{0}^{e} \cdot \mathbf{t}_{0}^{e} d \Gamma,
$$

where

$$
E_{k j}\left(\mathbf{v}_{0}^{e}\right)=\frac{1}{2}\left(\frac{\partial v_{0, k}^{e}}{\partial x_{j}}+\frac{\partial v_{0, j}^{e}}{\partial x_{k}}\right), \quad \mathbf{v}_{0}^{e}=\left(v_{0,1}^{e}, v_{0,2}^{e}, v_{0,3}^{e}\right),
$$

and $\mathbf{t}_{0}^{e}=\left(t_{0,1}^{e}, t_{0,2}^{e}, t_{0,3}^{e}\right)$ is the boundary traction due to the fields $\mathbf{v}_{0}^{e}$ and $p_{0}^{e}$, i.e.,

$$
t_{0, j}^{e}=T_{j k}\left(\mathbf{v}_{0}^{e}\right) n_{k}=\left(-p_{0}^{e} \delta_{j k}+2 E_{j k}\left(\mathbf{v}_{0}^{e}\right)\right) n_{k} .
$$

Note that in the identity (3.6) we have used the repeated-index summation convention. From now on, we will take into account this rule.

Using the equations (3.2), it can be proved that the fields $\mathbf{v}_{0}^{i}$ and $p_{0}^{i}$ satisfy the following identity (see [15], p. 15):

$$
2 \int_{D_{0}} E_{j k}\left(\mathbf{v}_{0}^{i}\right) E_{j k}\left(\mathbf{v}_{0}^{i}\right) d \mathbf{x}=\int_{\Gamma} \mathbf{v}_{0}^{i} \cdot \mathbf{t}_{0}^{i} d \Gamma,
$$

where

$$
E_{j k}\left(\mathbf{v}_{0}^{i}\right)=\frac{1}{2}\left(\frac{\partial v_{0, j}^{i}}{\partial x_{k}}+\frac{\partial v_{0, k}^{i}}{\partial x_{j}}\right), \quad \mathbf{v}_{0}^{i}=\left(v_{0,1}^{i}, v_{0,2}^{i}, v_{0,3}^{i}\right),
$$

and $\mathbf{t}_{0}^{i}$ is the boundary traction due to the fields $\mathbf{v}_{0}^{i}$ and $p_{0}^{i}$, i.e.,

$$
\mathbf{t}_{0}^{i}=\left(t_{0,1}^{i}, t_{0,2}^{i}, t_{0,3}^{i}\right), \quad t_{0, j}^{i}=T_{j k}\left(\mathbf{v}_{0}^{i}\right) n_{k}, \quad T_{j k}\left(\mathbf{v}_{0}^{i}\right)=\left(-p_{0}^{i} \delta_{j k}+2 E_{j k}\left(\mathbf{v}_{0}^{i}\right)\right) n_{k} .
$$

In view of the relations (3.3), (3.4), (3.6) and (3.7) we get the identity

$$
\int_{D_{e}}\left(\chi^{2}\left|\mathbf{v}_{0}^{e}\right|^{2}+2 E_{j k}\left(\mathbf{v}_{0}^{e}\right) E_{j k}\left(\mathbf{v}_{0}^{e}\right)\right) d \mathbf{x}=-2 \int_{D_{0}} E_{j k}\left(\mathbf{v}_{0}^{i}\right) E_{j k}\left(\mathbf{v}_{0}^{i}\right) d \mathbf{x} .
$$

Since the left-hand side of this identity is non-negative and the right-hand side is less than or equal to zero it follows that

$$
\int_{D_{e}}\left(\chi^{2}\left|\mathbf{v}_{0}^{e}\right|^{2}+2 E_{j k}\left(\mathbf{v}_{0}^{e}\right) E_{j k}\left(\mathbf{v}_{0}^{e}\right)\right) d \mathbf{x}=0, \quad \int_{D_{0}} E_{j k}\left(\mathbf{v}_{0}^{i}\right) E_{j k}\left(\mathbf{v}_{0}^{i}\right) d \mathbf{x}=0,
$$

and hence

$$
\begin{gathered}
\mathbf{v}_{0}^{e}=\mathbf{0} \text { in } D_{e}, \\
E_{j k}\left(\mathbf{v}_{0}^{i}\right)=0 \text { in } D_{0}, \quad i=1, \ldots, n .
\end{gathered}
$$


From (3.1) and (3.10) it follows that $\nabla p_{0}^{e}=\mathbf{0}$ in $D_{e}$, and the vanishing condition of $p_{0}$ at infinity yields that $p_{0}^{e}=0$ in $D_{e}$. Hence we have

$$
\mathbf{v}_{0}^{e}=\mathbf{0}, \quad p_{0}^{e}=0 \text { in } D_{e} .
$$

Now, using the condition (3.3), the property (3.10), and the uniqueness of the classical solution to the Stokes system in a bounded domain (see [15], Theorem 1.3.3), we deduce that

$$
\mathbf{v}_{0}^{i}=\mathbf{0}, \quad p_{0}^{i}=c \text { in } D_{0},
$$

where $c \in \mathbb{R}$.

On the other hand, from the properties (3.12) we find that

$$
\mathbf{t}_{0}^{e}=\mathbf{0} \text { on } \Gamma,
$$

and, in view of the condition (3.4), it follows that

$$
\mathbf{t}_{0}^{i}=-c \mathbf{n}=\mathbf{0} \text { on } \Gamma
$$

i.e., $c=0$. Therefore, we have

$$
\mathbf{v}_{0}^{e}=\mathbf{0}, \quad p_{0}^{e}=0 \text { in } D_{0} .
$$

The relations (3.12) and (3.15) yield that

$$
\left(\mathbf{v}_{1}^{e}, p_{1}^{e}\right)=\left(\mathbf{v}_{2}^{e}, p_{2}^{e}\right) \text { in } D_{e}, \quad\left(\mathbf{v}_{1}^{i}, p_{1}^{i}\right)=\left(\mathbf{v}_{2}^{i}, p_{2}^{i}\right) \text { in } D_{0},
$$

i.e., the desired uniqueness result. This completes the proof of Theorem 3.1.

4. The theory of hydrodynamic potentials. In this section we give a brief description of the main properties of the potential theory for the system of equations (2.6) and (2.7) and respectively for the Stokes system of equations (2.8) and (2.9).

4.1. The fundamental solution for the system of equations (2.6) and (2.7). Let us denote by $\left(\mathcal{G}^{\chi^{2}}\left(\mathcal{G}_{j k}^{\chi^{2}}\right), \Pi^{\chi^{2}}\left(\Pi_{j}^{\chi^{2}}\right)\right)$ the fundamental solution of the system of equations (2.6) and (2.7), i.e.,

$$
\begin{gathered}
\frac{\partial \mathcal{G}_{j k}^{\chi^{2}}(\mathbf{x}-\mathbf{y})}{\partial x_{j}}=0 \\
-\frac{\partial \Pi_{j}^{\chi^{2}}(\mathbf{x}-\mathbf{y})}{\partial x_{k}}+\left(\nabla_{\mathbf{x}}^{2}-\chi^{2}\right) \mathcal{G}_{k j}^{\chi^{2}}(\mathbf{x}-\mathbf{y})=-8 \pi \delta_{k j} \delta(\mathbf{x}-\mathbf{y}),
\end{gathered}
$$

where $\delta(\mathbf{x}-\mathbf{y})$ is the delta function or Dirac distribution in $\mathbb{R}^{3}$. Also $\nabla_{\mathbf{x}}$ means the gradient operator with respect to the point $\mathbf{x}$.

The functions $\mathcal{G}_{j k}^{\chi^{2}}$ and $\Pi_{j}^{\chi^{2}}$ are given by (see e.g. [15], p. 81)

$$
\begin{gathered}
\mathcal{G}_{j k}^{\chi^{2}}(\mathbf{x}-\mathbf{y})=\frac{\delta_{j k}}{|\mathbf{x}-\mathbf{y}|} A_{1}(\chi|\mathbf{x}-\mathbf{y}|)+\frac{\left(x_{j}-y_{j}\right)\left(x_{k}-y_{k}\right)}{|\mathbf{x}-\mathbf{y}|^{3}} A_{2}(\chi|\mathbf{x}-\mathbf{y}|), \\
\Pi_{j}^{\chi^{2}}(\mathbf{x}-\mathbf{y})=2 \frac{x_{j}-y_{j}}{|\mathbf{x}-\mathbf{y}|^{3}}
\end{gathered}
$$


where

$$
\begin{aligned}
& A_{1}(z)=2 e^{-z}\left(1+z^{-1}+z^{-2}\right)-2 z^{-2} \\
& A_{2}(z)=-2 e^{-z}\left(1+3 z^{-1}+3 z^{-2}\right)+6 z^{-2}
\end{aligned}
$$

Let $\mathbf{S}^{\chi^{2}}\left(S_{i j k}^{\chi^{2}}\right)$ be the stress tensor associated to the Green function $\mathcal{G}^{\chi^{2}}$, i.e.,

$$
\mathrm{S}_{i j k}^{\chi^{2}}(\mathbf{x}-\mathbf{y})=-\Pi_{j}^{\chi^{2}}(\mathbf{x}-\mathbf{y}) \delta_{j k}+\frac{\partial \mathcal{G}_{i j}^{\chi^{2}}(\mathbf{x}-\mathbf{y})}{\delta x_{k}}+\frac{\partial \mathcal{G}_{k j}^{\chi^{2}}(\mathbf{x}-\mathbf{y})}{\partial x_{i}}
$$

The components of this tensor, $S_{i j k}^{\chi^{2}}$, have the following expressions (see e.g. [15], p. $82)$ :

$$
\begin{aligned}
& S_{i j k}^{\chi^{2}}(\mathbf{x}-\mathbf{y})=-2\left\{\delta_{i k} \frac{x_{j}-y_{j}}{|\mathbf{x}-\mathbf{y}|^{3}} D_{1}(\chi|\mathbf{x}-\mathbf{y}|)\right. \\
& +\left(\delta_{k j} \frac{x_{i}-y_{i}}{|\mathbf{x}-\mathbf{y}|^{3}}+\delta_{i j} \frac{x_{k}-y_{k}}{|\mathbf{x}-\mathbf{y}|^{3}}\right) D_{2}(\chi|\mathbf{x}-\mathbf{y}|) \\
& \left.+\frac{\left(x_{i}-y_{i}\right)\left(x_{j}-y_{j}\right)\left(x_{k}-y_{k}\right)}{|\mathbf{x}-\mathbf{y}|^{5}} D_{3}(\chi|\mathbf{x}-\mathbf{y}|)\right\}
\end{aligned}
$$

where

$$
\begin{aligned}
& D_{1}(z)=2 e^{-z}\left(1+3 z^{-1}+3 z^{-2}\right)-6 z^{-2}+1 \\
& D_{2}(z)=e^{-z}\left(z+3+6 z^{-1}+6 z^{-2}\right)-6 z^{-2} \\
& D_{3}(z)=e^{-z}\left(-2 z-12-30 z^{-1}-30 z^{-2}\right)+30 z^{-2}
\end{aligned}
$$

Let $\boldsymbol{\Lambda}^{\chi^{2}}\left(\Lambda_{j k}^{\chi^{2}}\right)$ be the pressure tensor associated to the stress tensor $\mathbf{S}^{\chi^{2}}\left(S_{j k l}^{\chi^{2}}\right)$. These tensors determine a fundamental solution to the system of equations (2.6) and (2.7) in $\mathbb{R}^{3}$, i.e.,

$$
\begin{gathered}
\frac{\partial S_{j k l}^{\chi^{2}}(\mathbf{y}-\mathbf{x})}{\partial x_{k}}=0 \text { for } \mathbf{x} \neq \mathbf{y} \\
-\frac{\partial \Lambda_{j l}^{\chi^{2}}(\mathbf{x}-\mathbf{y})}{\partial x_{k}}+\left(\nabla_{\mathbf{x}}^{2}-\chi^{2}\right) S_{j k l}^{\chi^{2}}(\mathbf{y}-\mathbf{x})=0 \text { for } \mathbf{x} \neq \mathbf{y} .
\end{gathered}
$$

We have (see e.g. [15], pp. 190, 196)

$$
\Lambda_{i k}^{\chi^{2}}(\mathbf{x}-\mathbf{y})=2 \frac{\delta_{i k}}{|\mathbf{x}-\mathbf{y}|^{3}}\left(\mathcal{R}^{2}-2\right)+12 \frac{\left(x_{i}-y_{i}\right)\left(x_{k}-y_{k}\right)}{|\mathbf{x}-\mathbf{y}|^{5}},
$$

where $\mathcal{R}=\chi|\mathbf{x}-\mathbf{y}|$.

4.2. The fundamental solution of the Stokes system. Let $\left(\mathcal{G}\left(\mathcal{G}_{j k}\right), \Pi^{S}\left(\Pi_{j}^{S}\right)\right)$ be the fundamental solution of the Stokes system in $\mathbb{R}^{3}$, i.e.,

$$
\begin{gathered}
\frac{\partial \mathcal{G}_{j k}(\mathbf{x}-\mathbf{y})}{\partial x_{j}}=0 \\
-\frac{\partial \Pi_{k}^{S}(\mathbf{x}-\mathbf{y})}{\partial x_{j}}+\nabla_{\mathbf{x}}^{2} \mathcal{G}_{j k}(\mathbf{x}-\mathbf{y})=-8 \pi \delta_{k j} \delta(\mathbf{x}-\mathbf{y}) .
\end{gathered}
$$


We have (see e.g. [18], p. 50; [15], p. 38)

$$
\begin{gathered}
\mathcal{G}_{j k}(\mathbf{x}-\mathbf{y})=\frac{\delta_{k j}}{|\mathbf{x}-\mathbf{y}|}+\frac{\left(x_{j}-y_{j}\right)\left(x_{k}-y_{k}\right)}{|\mathbf{x}-\mathbf{y}|^{3}}, \\
\Pi_{j}^{S}(\mathbf{x}-\mathbf{y})=2 \frac{x_{j}-y_{j}}{|\mathbf{x}-\mathbf{y}|^{3}}
\end{gathered}
$$

Also, let $\mathbf{S}^{S}\left(S_{i j k}^{S}\right)$ be the stress tensor associated to the Green function $\mathcal{G}\left(\mathcal{G}_{j k}\right)$. Note that

$$
\begin{aligned}
\mathrm{S}_{i j k}^{S}(\mathbf{x}-\mathbf{y}) & =-\Pi_{j}^{S}(\mathbf{x}-\mathbf{y}) \delta_{j k}+\frac{\partial \mathcal{G}_{i j}(\mathbf{x}-\mathbf{y})}{\delta x_{k}}+\frac{\partial \mathcal{G}_{k j}(\mathbf{x}-\mathbf{y})}{\partial x_{i}} \\
& =-6 \frac{\left(x_{i}-y_{i}\right)\left(x_{j}-y_{j}\right)\left(x_{k}-y_{k}\right)}{|\mathbf{x}-\mathbf{y}|^{5}}
\end{aligned}
$$

For further arguments we denote by $\boldsymbol{\Lambda}^{S}\left(\Lambda_{i k}^{S}\right)$ the pressure tensor corresponding to the stress tensor $\mathbf{S}^{S}\left(S_{i j k}^{S}\right)$. The pair $\left(\mathbf{S}^{S}, \boldsymbol{\Lambda}^{S}\right)$ determine a fundamental solution to the Stokes system in the sense that

$$
\begin{gathered}
\frac{\partial S_{i j k}^{S}(\mathbf{y}-\mathbf{x})}{\partial x_{j}}=0 \text { for } \mathbf{x} \neq \mathbf{y} \\
-\frac{\partial \Lambda_{i k}^{S}(\mathbf{x}-\mathbf{y})}{\partial x_{j}}+\nabla_{\mathbf{x}}^{2} S_{i j k}^{S}(\mathbf{y}-\mathbf{x})=0 \text { for } \mathbf{x} \neq \mathbf{y}
\end{gathered}
$$

The pressure tensor $\boldsymbol{\Lambda}^{S}$ has the following components (see e.g. [15], pp. 128, 132):

$$
\Lambda_{i k}^{S}(\mathbf{x}-\mathbf{y})=4\left(-\frac{\delta_{i k}}{|\mathbf{x}-\mathbf{y}|^{3}}+3 \frac{\left(x_{i}-y_{i}\right)\left(x_{k}-y_{k}\right)}{|\mathbf{x}-\mathbf{y}|^{5}}\right) .
$$

4.3. The potential theory for the Stokes system. Let us consider the vector functions $\mathbf{g}=\left(g_{1}, g_{2}, g_{3}\right)$ and $\mathbf{h}=\left(h_{1}, h_{2}, h_{3}\right)$ such that $\mathbf{g}, \mathbf{h} \in C^{0}(\Gamma)$. The hydrodynamic singlelayer potential with density $\mathbf{g}$ is the vector function $\mathbf{V}(\cdot, \mathbf{g})$ given by

$$
\mathbf{V}(\mathbf{x}, \mathbf{g})=\frac{1}{8 \pi} \int_{\Gamma} \mathcal{G}(\mathbf{x}-\mathbf{y}) \cdot \mathbf{g}(\mathbf{y}) d \Gamma(\mathbf{y}), \quad \mathbf{x} \in \mathbb{R}^{3} \backslash \Gamma .
$$

The hydrodynamic double-layer potential with density $\mathbf{h}$ is the vector function $\mathbf{W}(\cdot, \mathbf{h})$ with the following components:

$$
W_{j}(\mathbf{x}, \mathbf{h})=\frac{1}{8 \pi} \int_{\Gamma} K_{i j}^{S}(\mathbf{y}, \mathbf{x}) h_{i}(\mathbf{y}) d \Gamma(\mathbf{y}), \quad \mathbf{x} \in \mathbb{R}^{3} \backslash \Gamma,
$$

where

$$
K_{i j}^{S}(\mathbf{y}, \mathbf{x})=S_{i j k}^{S}(\mathbf{y}-\mathbf{x}) n_{k}(\mathbf{y})
$$

Also, let $P^{s}(\cdot, \mathbf{g})$ and $P^{d}(\cdot, \mathbf{h})$ be the functions given by

$$
\begin{gathered}
P^{s}(\mathbf{x}, \mathbf{g})=\frac{1}{8 \pi} \int_{\Gamma} \Pi_{i}^{S}(\mathbf{x}-\mathbf{y}) g_{i}(\mathbf{y}) d \Gamma(\mathbf{y}), \quad \mathbf{x} \in \mathbb{R}^{3} \backslash \Gamma \\
P^{d}(\mathbf{x}, \mathbf{h})=\frac{1}{8 \pi} \int_{\Gamma} \Lambda_{i k}^{S}(\mathbf{x}-\mathbf{y}) n_{k}(\mathbf{y}) h_{i}(\mathbf{y}) d \Gamma(\mathbf{y}), \quad \mathbf{x} \in \mathbb{R}^{3} \backslash \Gamma .
\end{gathered}
$$


According to the equations (4.11), (4.12), (4.16) and (4.17), we deduce that each of the pairs $\left(\mathbf{V}(\cdot, \mathbf{g}), P^{s}(\cdot, \mathbf{g})\right)$ and $\left(\mathbf{W}(\cdot, \mathbf{h}), P^{d}(\cdot, \mathbf{h})\right)$ is a classical solution to the Stokes system in both regions $D_{0}$ and $D_{e}$, i.e.,

$$
\begin{aligned}
& \nabla \cdot \mathbf{V}(\cdot, \mathbf{g})=0, \quad-\nabla P^{s}(\cdot, \mathbf{g})+\nabla^{2} \mathbf{V}(\cdot, \mathbf{g})=\mathbf{0} \\
& \nabla \cdot \mathbf{W}(\cdot, \mathbf{h})=0, \quad-\nabla P^{d}(\cdot, \mathbf{h})+\nabla^{2} \mathbf{W}(\cdot, \mathbf{h})=\mathbf{0}
\end{aligned}
$$

in $D_{0}$ and $D_{e}$ respectively.

The single- and double-layer potentials behave at infinity as follows:

$$
\begin{gathered}
\mathbf{V}(\mathbf{x}, \mathbf{g})=\mathcal{O}\left(|\mathbf{x}|^{-1}\right) \text { as }|\mathbf{x}| \rightarrow \infty \\
P^{s}(\mathbf{x}, \mathbf{h})=\mathcal{O}\left(|\mathbf{x}|^{-2}\right) \text { as }|\mathbf{x}| \rightarrow \infty . \\
\mathbf{W}(\mathbf{x}, \mathbf{h})=\mathcal{O}\left(|\mathbf{x}|^{-2}\right), \quad P^{d}(\mathbf{x}, \mathbf{h})=\mathcal{O}\left(|\mathbf{x}|^{-3}\right) \text { as }|\mathbf{x}| \rightarrow \infty .
\end{gathered}
$$

Let $\mathbf{T}(\mathbf{V})$ be the stress tensor field due to the single-layer potential $\mathbf{V}(\cdot, \mathbf{g})$. According to the equations (4.15) we find that

$$
\begin{aligned}
T_{i j}(\mathbf{V})(\mathbf{x}) & =-P^{s}(\mathbf{x}, \mathbf{g}) \delta_{i j}+\frac{\partial V_{i}(\mathbf{x}, \mathbf{g})}{\partial x_{j}}+\frac{\partial V_{j}(\mathbf{x}, \mathbf{g})}{\partial x_{i}} \\
& =\frac{1}{8 \pi} \int_{\Gamma} S_{i j k}^{S}(\mathbf{x}-\mathbf{y}) g_{i}(\mathbf{y}) d \Gamma(\mathbf{y}), \quad \mathbf{x} \in \mathbb{R}^{3} \backslash \Gamma .
\end{aligned}
$$

In addition, the normal stresses due to the single-layer potential $\mathbf{V}(\cdot, \mathbf{g})$ are defined in a neighbourhood $U$ of $\Gamma$ by the relation

$$
t_{j}(\mathbf{V})(\mathbf{x})=T_{j l}(\mathbf{V})(\mathbf{x}) n_{l}(\tilde{\mathbf{x}}), \quad \mathbf{x} \in U \backslash \Gamma, j=1,2,3,
$$

where $\tilde{\mathbf{x}}$ is the unique projection of $\mathbf{x} \in U$ onto $\Gamma$.

Let $w$ be a (scalar, vector, or tensor) field defined in a domain containing $\Gamma$. Then we denote by $w^{-}\left(\mathbf{x}_{0}\right)$ and $w^{+}\left(\mathbf{x}_{0}\right)$ the limiting values of $w$ in a point $\mathbf{x}_{0} \in \Gamma$, evaluated from $D_{0}$ and $D_{e}$ respectively.

The following theorem refers to the continuity behaviour of the hydrodynamic potentials across the boundary $\Gamma$ of the domain $D_{0}$ (see e.g. [15], Chapter 3):

Theorem 4.1. Let $\mathbf{g}$ and $\mathbf{h}$ be two continuous vector fields on $\Gamma$ and $\operatorname{let} \mathbf{V}(\cdot, \mathbf{g}), \mathbf{W}(\cdot, \mathbf{h})$ and $\mathbf{t}(\mathbf{V})$ be the functions given by the relations (4.19), (4.20) and (4.30). Then for any $\mathbf{x}_{0} \in \Gamma$ we have

$$
\begin{gathered}
\mathbf{V}^{+}\left(\mathbf{x}_{0}, \mathbf{g}\right)=\mathbf{V}^{-}\left(\mathbf{x}_{0}, \mathbf{g}\right)=\mathbf{V}\left(\mathbf{x}_{0}, \mathbf{g}\right), \\
W_{j}^{+}\left(\mathbf{x}_{0}, \mathbf{h}\right)=\frac{1}{2} h_{j}\left(\mathbf{x}_{0}\right)+\frac{1}{8 \pi} \int_{\Gamma} K_{i j}^{S}\left(\mathbf{y}, \mathbf{x}_{0}\right) h_{i}(\mathbf{y}) d \Gamma(\mathbf{y}), \\
W_{j}^{-}\left(\mathbf{x}_{0}, \mathbf{h}\right)=-\frac{1}{2} h_{j}\left(\mathbf{x}_{0}\right)+\frac{1}{8 \pi} \int_{\Gamma} K_{i j}^{S}\left(\mathbf{y}, \mathbf{x}_{0}\right) h_{i}(\mathbf{y}) d \Gamma(\mathbf{y}), \\
t_{j}^{+}(\mathbf{V})\left(\mathbf{x}_{0}\right)=-\frac{1}{2} g_{j}\left(\mathbf{x}_{0}\right)+\frac{1}{8 \pi} \int_{\Gamma} K_{j i}^{S}\left(\mathbf{x}_{0}, \mathbf{y}\right) g_{i}(\mathbf{y}) d \Gamma(\mathbf{y}), \\
t_{j}^{-}(\mathbf{V})\left(\mathbf{x}_{0}\right)=\frac{1}{2} g_{j}\left(\mathbf{x}_{0}\right)+\frac{1}{8 \pi} \int_{\Gamma} K_{j i}^{S}\left(\mathbf{x}_{0}, \mathbf{y}\right) g_{i}(\mathbf{y}) d \Gamma(\mathbf{y}),
\end{gathered}
$$

where all double-layer integrals are improper integrals, with weakly singular kernels. 
Let us now consider the single- and double-layer integral operators

$$
\mathcal{V}: C^{0}(\Gamma) \rightarrow C^{0}(\Gamma), \quad \mathbf{K}^{d}: C^{0}(\Gamma) \rightarrow C^{0}(\Gamma)
$$

given by

$$
(\mathcal{V} \mathbf{g})\left(\mathbf{x}_{0}\right)=\mathbf{V}\left(\mathbf{x}_{0}, \mathbf{g}\right), \quad\left(\mathbf{K}^{d} \mathbf{h}\right)_{j}\left(\mathbf{x}_{0}\right)=\frac{1}{8 \pi} \int_{\Gamma} K_{i j}^{S}\left(\mathbf{y}, \mathbf{x}_{0}\right) h_{i}(\mathbf{y}) d \Gamma(\mathbf{y})
$$

for $\mathbf{x}_{0} \in \Gamma$ and all $\mathbf{g}, \mathbf{h} \in C^{0}(\Gamma)$.

Since $\Gamma$ is a closed Lyapunov surface it follows that the kernels of the integral operators $\mathcal{V}$ and $\mathbf{K}^{d}, \mathcal{G}(\mathbf{x}-\mathbf{y})$ and $\mathbf{K}^{S}(\mathbf{y}-\mathbf{x})$, are weakly singular, and hence these operators are compact from $C^{0}(\Gamma)$ into $C^{0}(\Gamma)$.

Let $\langle\cdot, \cdot\rangle: L^{2}(\Gamma) \times L^{2}(\Gamma) \rightarrow \mathbb{R}$ be the inner product given by

$$
\langle\mathbf{g}, \mathbf{h}\rangle=\int_{\Gamma} \mathbf{g}(\mathbf{y}) \cdot \mathbf{h}(\mathbf{y}) d \Gamma(\mathbf{y}), \quad \mathbf{g}, \mathbf{h} \in L^{2}(\Gamma),
$$

and let $\mathbf{K}^{d *}: C^{0}(\Gamma) \rightarrow C^{0}(\Gamma)$ be the adjoint of the double-layer integral operator $\mathbf{K}^{d}$ with respect to this inner product. Straightforward computation yields that

$$
\left(\mathbf{K}^{d *} \mathbf{g}\right)_{j}(\mathbf{x})=\frac{1}{8 \pi} \int_{\Gamma} K_{j i}^{S}(\mathbf{x}, \mathbf{y}) g_{i}(\mathbf{y}) d \Gamma(\mathbf{y}), \quad \mathbf{g} \in C^{0}(\Gamma), \mathbf{x} \in \Gamma,
$$

$j=1, \ldots, n$. Note that $\mathbf{K}^{d *}$ is also compact from $C^{0}(\Gamma)$ into $C^{0}(\Gamma)$.

Assume that $0<\gamma \leq 1$. For the next arguments, we denote by $C^{\gamma}(\Gamma)$ the set of Hölder continuous real-valued functions on $\Gamma$ with exponent $\gamma$. Also let $C^{k, \gamma}(\Gamma)$ be the space of those real-valued functions that are $k$-times continuously differentiable and whose $k^{t h}$-partial derivatives are Hölder continuous on $\Gamma$ with exponent $\gamma$. For simplicity, the spaces $\left(C^{\gamma}(\Gamma)\right)^{3}$ and $\left(C^{k, \gamma}(\Gamma)\right)^{3}$ of corresponding vector functions will be simply denoted by $C^{\gamma}(\Gamma)$ and $C^{k, \gamma}(\Gamma)$, but we always make a distinction between the two different cases. Note that $C^{k, \gamma}(\Gamma)$ is a Banach space (see e.g. [6], p. 241).

In addition to the previous properties, we have the following results related to the single- and double-layer integral operators $\mathcal{V}$ and $\mathbf{K}^{d}$ (see e.g. [7], [20]):

TheOREM 4.2. Let us assume that $\Gamma$ is a closed Lyapunov surface of class $C^{1, \alpha}, \alpha \in(0,1]$, and let $\lambda \in(0, \alpha)$. Then:

- The single-layer integral operator $\mathcal{V}$ is continuous from $C^{\lambda}(\Gamma)$ into $C^{1, \lambda}(\Gamma)$.

- The double-layer integral operator $\mathbf{K}^{d}$ is continuous from $C^{1, \lambda}(\Gamma)$ into $C^{1, \lambda}(\Gamma)$.

On the other hand, we have the following property:

- If $\Gamma \in C^{2, \alpha}, \alpha \in(0,1]$, and $\Phi \in C^{1, \gamma}(\Gamma), \gamma \in(0,1)$, then there exist the limiting values of the boundary traction due to the double-layer potential $\mathbf{W}(\cdot, \Phi)$ on both sides of $\Gamma, \mathbf{T}^{+}(\mathbf{W}(\Phi))$ and $\mathbf{T}^{-}(\mathbf{W}(\Phi))$, and these limiting values are equal, i.e.,

$$
\mathbf{T}^{+}(\mathbf{W}(\Phi))=\mathbf{T}^{-}(\mathbf{W}(\Phi)) \equiv \mathbf{T}(\mathbf{W}(\Phi)) \text { on } \Gamma .
$$

The equality (4.39) expresses the continuity property of the boundary traction due to the double-layer potential $\mathbf{W}(\cdot, \Phi)$ across the boundary $\Gamma$, when the density $\Phi$ belongs to the class $C^{1, \gamma}(\Gamma), \gamma \in(0,1)$, and is similar to the Lyapunov-Tauber Theorem from the classical potential theory (see e.g. [29], p. 163; [15], p. 151). 
4.4. The potential theory for the system of equations (2.6) and (2.7). Let us now refer to the system of equations (2.6) and (2.7) and let $\mathbf{g}$ and $\mathbf{h}$ be two continuous vector functions on $\Gamma$. Then the single- and double-layer potentials, $\mathbf{V}_{\chi^{2}, 3}(\cdot, \mathbf{g}) \mathbf{W}_{\chi^{2}, 3}(\cdot, \mathbf{h})$, associated to this system, are given by

$$
\begin{array}{r}
\mathbf{V}_{\chi^{2}, 3}(\mathbf{x}, \mathbf{g})=\frac{1}{8 \pi} \int_{\Gamma} \mathcal{G}^{\chi^{2}}(\mathbf{x}-\mathbf{y}) \cdot \mathbf{g}(\mathbf{y}) d \Gamma(\mathbf{y}), \quad \mathbf{x} \in \mathbb{R}^{3} \backslash \Gamma, \\
\left(\mathbf{W}_{\chi^{2}, 3}\right)_{j}(\mathbf{x}, \mathbf{h})=\frac{1}{8 \pi} \int_{\Gamma} S_{i j k}^{\chi^{2}}(\mathbf{y}-\mathbf{x}) n_{k}(\mathbf{y}) h_{i}(\mathbf{y}) d \Gamma(\mathbf{y}), \quad \mathbf{x} \in \mathbb{R}^{3} \backslash \Gamma .
\end{array}
$$

We also consider the functions $P_{\chi^{2}, 3}^{s}(\cdot, \mathbf{g})$ and $P_{\chi^{2}, 3}^{d}(\cdot, \mathbf{h})$ given by

$$
\begin{gathered}
P_{\chi^{2}, 3}^{s}(\mathbf{x}, \mathbf{g})=\frac{1}{8 \pi} \int_{\Gamma} \Pi_{i}^{\chi^{2}}(\mathbf{x}-\mathbf{y}) g_{i}(\mathbf{y}) d \Gamma(\mathbf{y}), \quad \mathbf{x} \in \mathbb{R}^{3} \backslash \Gamma \\
P_{\chi^{2}, 3}^{d}(\mathbf{x}, \mathbf{h})=\frac{1}{8 \pi} \int_{\Gamma} \Lambda_{i k}^{\chi^{2}}(\mathbf{x}-\mathbf{y}) n_{k}(\mathbf{y}) h_{i}(\mathbf{y}) d \Gamma(\mathbf{y}), \quad \mathbf{x} \in \mathbb{R}^{3} \backslash \Gamma .
\end{gathered}
$$

Taking into account the equations (4.1), (4.2), (4.8) and (4.9) we deduce that each of the pairs $\left(\mathbf{V}_{\chi^{2}, 3}(\cdot, \mathbf{g}), P_{\chi^{2}, 3}^{s}(\cdot, \mathbf{g})\right)$ and $\left(\mathbf{W}_{\chi^{2}, 3}(\cdot, \mathbf{h}), P_{\chi^{2}, 3}^{d}(\cdot, \mathbf{h})\right)$ is a classical solution to the system of equations (2.6) and (2.7) in $\mathbb{R}^{3} \backslash \Gamma$, i.e.,

$$
\begin{gathered}
\nabla \cdot \mathbf{V}_{\chi^{2}, 3}(\cdot, \mathbf{g})=\mathbf{0}, \quad-\nabla P_{\chi^{2}, 3}^{s}(\cdot, \mathbf{g})+\left(\nabla^{2}-\chi^{2}\right) \mathbf{V}_{\chi^{2}, 3}(\cdot, \mathbf{g})=\mathbf{0}, \\
\nabla \cdot \mathbf{W}_{\chi^{2}, 3}(\cdot, \mathbf{h})=\mathbf{0}, \quad-\nabla P_{\chi^{2}, 3}^{d}(\cdot, \mathbf{h})+\left(\nabla^{2}-\chi^{2}\right) \mathbf{W}_{\chi^{2}, 3}(\cdot, \mathbf{h})=\mathbf{0}
\end{gathered}
$$

in $\mathbb{R}^{3} \backslash \Gamma$.

Let $\mathbf{H}_{\chi^{2}, 3}(\cdot, \mathbf{g})$ be the normal stresses provided by the single-layer potential $\mathbf{V}_{\chi^{2}, 3}(\cdot, \mathbf{g})$ defined in a neighbourhood $U \subset \mathbb{R}^{3}$ of $\Gamma$ by the relations

$$
\left(\mathbf{H}_{\chi^{2}, 3}\right)_{j}(\mathbf{x}, \mathbf{g})=\frac{1}{8 \pi} \int_{\Gamma} S_{j i k}^{\chi^{2}}(\mathbf{x}-\mathbf{y}) n_{k}(\tilde{\mathbf{x}}) g_{i}(\mathbf{y}) d \Gamma(\mathbf{y}), \quad \mathbf{x} \notin \Gamma,
$$

where $\tilde{\mathbf{x}}$ is the unique projection of $\mathbf{x} \in U$ onto $\Gamma$.

Let us introduce the kernel matrices $\mathbf{K}_{\chi^{2}, 3}(\mathbf{y}, \mathbf{x})$ and $\mathbf{D}_{\chi^{2}, 3}(\mathbf{x}, \mathbf{y})$ with the components

$$
\left(\mathbf{K}_{\chi^{2}, 3}\right)_{i j}(\mathbf{y}, \mathbf{x})=S_{i j k}^{\chi^{2}}(\mathbf{y}-\mathbf{x}) n_{k}(\mathbf{y}), \quad\left(\mathbf{D}_{\chi^{2}, 3}\right)_{i j}(\mathbf{x}, \mathbf{y})=S_{j i k}^{\chi^{2}}(\mathbf{x}-\mathbf{y}) n_{k}(\tilde{\mathbf{x}}),
$$

$i, j=1,2,3$. Therefore, the double-layer potential $\mathbf{W}_{\chi^{2}, 3}(\cdot, \mathbf{h})$ and the normal stress associated with the single-layer potential $\mathbf{H}_{\chi^{2}, 3}(\cdot, \mathbf{g})$ can be written in the form

$$
\begin{aligned}
& \mathbf{W}_{\chi^{2}, 3}(\mathbf{x}, \mathbf{h})=\frac{1}{3 \pi} \int_{\Gamma} \mathbf{h}(\mathbf{y}) \cdot \mathbf{K}_{\chi^{2}, 3}(\mathbf{y}, \mathbf{x}) d \Gamma(\mathbf{y}), \\
& \mathbf{H}_{\chi^{2}, 3}(\mathbf{x}, \mathbf{g})=\frac{1}{8 \pi} \int_{\Gamma} \mathbf{g}(\mathbf{y}) \cdot \mathbf{D}_{\chi^{2}, 3}(\mathbf{x}, \mathbf{y}) d \Gamma(\mathbf{y})
\end{aligned}
$$

for $\mathbf{x} \notin \Gamma$.

Furthermore, using the decomposition formulas

$$
\begin{gathered}
\mathcal{G}_{j i}^{\chi^{2}}(\mathbf{x}-\mathbf{y})=\mathcal{G}_{j i}(\mathbf{x}-\mathbf{y})+\mathcal{G}_{j i}^{c}(\mathbf{x}-\mathbf{y}) \\
S_{i j k}^{\chi^{2}}(\mathbf{y}-\mathbf{x}) n_{k}(\mathbf{y})=S_{i j k}^{S}(\mathbf{y}-\mathbf{x}) n_{k}(\mathbf{y})+S_{i j k}^{c}(\mathbf{y}-\mathbf{x}) n_{k}(\mathbf{y}),
\end{gathered}
$$


where the kernel $\mathcal{G}^{c}$ is continuous and $\mathbf{S}^{c}$ bounded, we deduce that the continuity behaviour of the functions $\mathbf{V}_{\chi^{2}, 3}(\cdot, \mathbf{g}), \mathbf{W}_{\chi^{2}, 3}(\cdot, \mathbf{h})$ and $\mathbf{H}_{\chi^{2}, 3}(\cdot, \mathbf{g})$ is determined only by the tensors $\mathcal{G}$ and $\mathbf{S}^{S}$, which correspond to the Stokes system. Therefore, we have the following result (see e.g. [15], p. 201):

TheOrem 4.3. Let $\mathbf{g}$ and $\mathbf{h}$ be two continuous vector functions on $\Gamma$ and let $\mathbf{V}_{\chi^{2}, 3}(\cdot, \mathbf{g})$, $\mathbf{W}_{\chi^{2}, 3}(\cdot, \mathbf{h})$ and $\mathbf{H}_{\chi^{2}, 3}(\cdot, \mathbf{g})$ be the functions given by the relations (4.40), (4.41) and (4.46). Then for any $\mathbf{x}_{0} \in \Gamma$

$$
\begin{gathered}
\mathbf{V}_{\chi^{2}, 3}^{+}\left(\mathbf{x}_{0}, \mathbf{g}\right)=\mathbf{V}_{\chi^{2}, 3}^{-}\left(\mathbf{x}_{0}, \mathbf{g}\right)=\mathbf{V}_{\chi^{2}, 3}\left(\mathbf{x}_{0}, \mathbf{g}\right) \\
\mathbf{W}_{\chi^{2}, 3}^{+}\left(\mathbf{x}_{0}, \mathbf{h}\right)-\mathbf{W}_{\chi^{2}, 3}^{*}\left(\mathbf{x}_{0}, \mathbf{h}\right)=\mathbf{h}\left(\mathbf{x}_{0}\right)=\mathbf{W}_{\chi^{2}, 3}^{*}\left(\mathbf{x}_{0}, h\right)-\mathbf{W}_{\chi^{2}, 3}^{-}\left(\mathbf{x}_{0}, \mathbf{h}\right) \\
\mathbf{H}_{\chi^{2}, 3}^{+}\left(\mathbf{x}_{0}, \mathbf{g}\right)-\mathbf{H}_{\chi^{2}, 3}^{*}\left(\mathbf{x}_{0}, \mathbf{g}\right)=-\mathbf{g}\left(\mathbf{x}_{0}\right)=\mathbf{H}_{\chi^{2}, 3}^{*}\left(\mathbf{x}_{0}, \mathbf{g}\right)-\mathbf{H}_{\chi^{2}, 3}^{-}\left(\mathbf{x}_{0}, \mathbf{g}\right)
\end{gathered}
$$

where

$$
\begin{aligned}
& \left(\mathbf{W}_{\chi^{2}, 3}^{*}\right)_{j}\left(\mathbf{x}_{0}, \mathbf{h}\right)=\frac{1}{8 \pi} \int_{\Gamma} S_{i j k}^{\chi^{2}}\left(\mathbf{y}-\mathbf{x}_{0}\right) n_{k}(\mathbf{y}) h_{i}(\mathbf{y}) d \Gamma(\mathbf{y}), \\
& \left(\mathbf{H}_{\chi^{2}, 3}^{*}\right)_{j}\left(\mathbf{x}_{0}, \mathbf{g}\right)=\frac{1}{8 \pi} \int_{\Gamma} S_{j i k}^{\chi^{2}}\left(\mathbf{x}_{0}-\mathbf{y}\right) n_{k}\left(\mathbf{x}_{0}\right) g_{i}(\mathbf{y}) d \Gamma(\mathbf{y}) .
\end{aligned}
$$

In addition, $\mathbf{V}_{\chi^{2}, 3}(\cdot, \mathbf{g}), \mathbf{W}_{\chi^{2}, 3}(\cdot, \mathbf{h}), P_{\chi^{2}, 3}^{s}(\cdot, \mathbf{g}), P_{\chi^{2}, 3}^{d}(\cdot, \mathbf{h})$ have the following decay behaviour at infinity:

$$
\begin{aligned}
\mathbf{V}_{\chi^{2}, 3}(\mathbf{x}, \mathbf{g})= & \mathcal{O}\left(|\mathbf{x}|^{-3}\right), \quad \mathbf{W}_{\chi^{2}, 3}(\mathbf{x}, \mathbf{h})=\mathcal{O}\left(|\mathbf{x}|^{-2}\right) \text { as }|\mathbf{x}| \rightarrow \infty \\
& P_{\chi^{2}, 3}^{s}(\mathbf{x}, \mathbf{g})=\mathcal{O}\left(|\mathbf{x}|^{-2}\right) \text { as }|\mathbf{x}| \rightarrow \infty \\
& P_{\chi^{2}, 3}^{d}(\mathbf{x}, \mathbf{h})=\mathcal{O}\left(|\mathbf{x}|^{-1}\right) \text { as }|\mathbf{x}| \rightarrow \infty
\end{aligned}
$$

Let $\mathcal{V}_{\chi^{2}, 3}: C^{0}(\Gamma) \rightarrow C^{0}(\Gamma)$ and $\mathbf{K}_{\chi^{2}, 3}^{d}: C^{0}(\Gamma) \rightarrow C^{0}(\Gamma)$ be the single- and double-layer integral operators given by

$$
\left(\mathcal{V}_{\chi^{2}, 3} \mathbf{g}\right)\left(\mathbf{x}_{0}\right)=\mathbf{V}_{\chi^{2}, 3}\left(\mathbf{x}_{0}, \mathbf{g}\right), \quad\left(\mathbf{K}_{\chi^{2}, 3}^{d} \mathbf{h}\right)\left(\mathbf{x}_{0}\right)=\mathbf{W}_{\chi^{2}, 3}^{*}\left(\mathbf{x}_{0}, \mathbf{h}\right),
$$

for $\mathbf{x}_{0} \in \Gamma$ and all $\mathbf{g}, \mathbf{h} \in C^{0}(\Gamma)$. Using the decomposition formulas (4.49) it follows that both kernels $\mathcal{G}^{\chi^{2}}(\mathbf{x}-\mathbf{y})$ and $\mathbf{K}_{\chi^{2}, 3}(\mathbf{y}, \mathbf{x})$ of these operators have a weakly singular behaviour. Consequently, both operators $\mathbf{V}_{\chi^{2}, 3}$ and $\mathbf{K}_{\chi^{2}, 3}^{d}$ are compact from $C^{0}(\Gamma)$ into $C^{0}(\Gamma)$. In addition, we have the following properties (see also [17], where there are mentioned mapping properties of these operators):

TheOREM 4.4. - If $\Gamma \in C^{2, \alpha}$ and $\lambda \in(0, \alpha), \alpha \in(0,1]$, then the new integral operators $\mathcal{V}^{c}: C^{\lambda}(\Gamma) \rightarrow C^{1, \lambda}(\Gamma)$ and $\mathbf{K}^{c}: C^{1, \lambda}(\Gamma) \rightarrow C^{1, \lambda}(\Gamma)$ given by

$$
\mathcal{V}^{c}=\mathcal{V}_{\chi^{2}, 3}-\mathcal{V}, \quad \mathbf{K}^{c}=\mathbf{K}_{\chi^{2}, 3}-\mathbf{K}
$$

are compact linear operators.

- If $\Gamma \in C^{2, \alpha}$ and $\Phi \in C^{1, \gamma}(\Gamma), \alpha \in(0,1], \gamma \in(0,1)$, then there exist the limiting values of the boundary traction due to the double-layer potential $\mathbf{W}_{\chi^{2}, 3}(\cdot, \Phi)$ on both sides of $\Gamma, \mathbf{T}^{+}\left(\mathbf{W}_{\chi^{2}, 3}(\Phi)\right)$ and $\mathbf{T}^{-}\left(\mathbf{W}_{\chi^{2}, 3}(\Phi)\right)$, and they are equal, i.e.,

$$
\mathbf{T}^{+}\left(\mathbf{W}_{\chi^{2}, 3}(\Phi)\right)=\mathbf{T}^{-}\left(\mathbf{W}_{\chi^{2}, 3}(\Phi)\right) \equiv \mathbf{T}\left(\mathbf{W}_{\chi^{2}, 3}(\Phi)\right) \text { on } \Gamma \text {. }
$$


Proof. Taking into account the expressions of the complementary kernels $\mathcal{G}_{j i}^{c}(\mathbf{x}-\mathbf{y})$ and $S_{i j k}^{c}(\mathbf{y}-\mathbf{x}) n_{k}(\mathbf{y})$ (see the relations (4.3)-(4.7), (4.13) and (4.15)) it can be proved that the integral operators

$$
\mathcal{V}^{c}: C^{\lambda}(\Gamma) \rightarrow C^{2, \lambda}(\Gamma), \quad \mathbf{K}^{c}: C^{1, \lambda}(\Gamma) \rightarrow C^{2, \lambda}(\Gamma)
$$

are continuous. In addition, the space $C^{2, \lambda}(\Gamma)$ is compactly embedded into $C^{1, \lambda}(\Gamma)$ (see e.g. [42]). Therefore, the operator $\mathcal{V}^{c}: C^{\lambda}(\Gamma) \rightarrow C^{1, \lambda}(\Gamma)$ is compact as the product of the continuous operator $\mathcal{V}^{c}: C^{\lambda}(\Gamma) \rightarrow C^{2, \lambda}(\Gamma)$ with the compact embedding operator from $C^{2, \lambda}(\Gamma)$ into $C^{1, \lambda}(\Gamma)$. With similar kinds of arguments it follows that the operator $\mathbf{K}^{c}: C^{1, \lambda}(\Gamma) \rightarrow C^{1, \lambda}(\Gamma)$ is also compact.

The proof of the property (4.58) can be consulted in [39].

Let $\mathcal{H}_{\chi^{2}, 3}$ be the adjoint of the double-layer integral operator $\mathbf{K}_{\chi^{2}, 3}^{d}$ with respect to the inner product $\langle\cdot, \cdot\rangle$ given by the relation (4.37). Then we have

$$
\left(\mathcal{H}_{\chi^{2}, 3} \mathbf{g}\right)\left(\mathbf{x}_{0}\right)=\mathbf{H}_{\chi^{2}, 3}^{*}\left(\mathbf{x}_{0}, \mathbf{g}\right), \quad \mathbf{x}_{0} \in \Gamma, \mathbf{g} \in C^{0}(\Gamma)
$$

5. The boundary integral formulation and the existence result of the solution. Let $\lambda$ be an arbitrary number in the interval $(0, \alpha)$.

In order to prove that the boundary value problem (2.6)-(2.12) has a unique classical solution, we consider the following boundary integral representations:

$$
\begin{aligned}
v_{k}^{e}(\mathbf{x}) & =U_{k}^{\infty}+\frac{1}{8 \pi} \int_{\Gamma} K_{j k}^{\chi^{2}}(\mathbf{y}, \mathbf{x}) \phi_{j}(\mathbf{y}) d \Gamma(\mathbf{y}) \\
& +\frac{1}{8 \pi} \int_{\Gamma} \mathcal{G}_{k j}^{\chi^{2}}(\mathbf{x}-\mathbf{y}) h_{j}(\mathbf{y}) d \Gamma(\mathbf{y}), \quad \mathbf{x} \in D_{e} \\
p^{e}(\mathbf{x})= & p^{\infty}(\mathbf{x})+\frac{1}{8 \pi} \int_{\Gamma} \Lambda_{j k}^{\chi^{2}}(\mathbf{x}-\mathbf{y}) n_{k}(\mathbf{y}) \phi_{j}(\mathbf{y}) d \Gamma(\mathbf{y}) \\
+ & \frac{1}{8 \pi} \int_{\Gamma} \Pi_{j}^{\chi^{2}}(\mathbf{x}-\mathbf{y}) h_{j}(\mathbf{y}) d \Gamma(\mathbf{y}), \quad \mathbf{x} \in D_{e},
\end{aligned}
$$

and

$$
\begin{aligned}
v_{k}^{i}(\mathbf{x}) & =\frac{1}{8 \pi} \int_{\Gamma} K_{j k}^{S}(\mathbf{y}, \mathbf{x}) \phi_{j}(\mathbf{y}) d \Gamma(\mathbf{y}) \\
& +\frac{1}{8 \pi} \int_{\Gamma} \mathcal{G}_{k j}(\mathbf{x}-\mathbf{y}) h_{j}(\mathbf{y}) d \Gamma(\mathbf{y}), \quad \mathbf{x} \in D_{0}, \\
p^{i}(\mathbf{x}) & =\frac{1}{8 \pi} \int_{\Gamma} \Lambda_{j k}^{S}(\mathbf{x}-\mathbf{y}) n_{k}(\mathbf{y}) \phi_{j}(\mathbf{y}) d \Gamma(\mathbf{y}) \\
& +\frac{1}{8 \pi} \int_{\Gamma} \Pi_{j}^{S}(\mathbf{x}-\mathbf{y}) h_{j}(\mathbf{y}) d \Gamma(\mathbf{y}), \quad \mathbf{x} \in D_{0},
\end{aligned}
$$

where $\Phi \in C^{1, \lambda}(\Gamma)$ and $\mathbf{h} \in C^{\lambda}(\Gamma)$ are unknown vector densities.

Therefore, we try to determine the external velocity field $\mathbf{v}^{e}-\mathbf{U}^{\infty}$ as a combination between a double-layer potential and a single-layer potential, $\mathbf{W}_{\chi^{2}, 3}(\cdot, \Phi)$ and $\mathbf{V}_{\chi^{2}, 3}(\cdot, \mathbf{h})$, each of them corresponding to the system of the continuity and Brinkman equations and having the unknown densities $\Phi=\left(\phi_{1}, \phi_{2}, \phi_{3}\right)$ and $\mathbf{h}=\left(h_{1}, h_{2}, h_{3}\right)$ respectively. Also, the velocity field $\mathbf{v}^{i}$ of the inner flow is thought of as a combination between a double-layer 
potential and a single-layer potential, $\mathbf{W}(\cdot, \Phi)$ and $\mathbf{V}(\cdot, \mathbf{h})$, each of them corresponding to the Stokes system.

According to the equations (4.24), (4.25), (4.44) and (4.45) it follows that the fields $\mathbf{v}^{e}$ and $p^{e}$ satisfy the system of equations (2.6) and (2.7). Similarly, the fields $\mathbf{v}^{i}$ and $p^{i}$ satisfy the equations (2.8) and (2.9). In addition, the formulas (4.54) and (4.55) yield that the far field conditions (2.12) are satisfied.

On the other hand, using the continuity behaviour of single- and double-layer potentials across the boundary $\Gamma$, i.e. the properties (4.31)-(4.33), (4.50) and (4.51), we find that

$$
\begin{aligned}
v_{k}^{e+}\left(\mathbf{x}_{0}\right)= & U_{k}^{\infty}+\frac{1}{2} \phi_{k}\left(\mathbf{x}_{0}\right)+\frac{1}{8 \pi} \int_{\Gamma} K_{j k}^{\chi^{2}}\left(\mathbf{y}, \mathbf{x}_{0}\right) \phi_{j}(\mathbf{y}) d \Gamma(\mathbf{y}) \\
+ & \frac{1}{8 \pi} \int_{\Gamma} \mathcal{G}_{k j}^{\chi^{2}}\left(\mathbf{x}_{0}-\mathbf{y}\right) h_{j}(\mathbf{y}) d \Gamma(\mathbf{y}), \quad \mathbf{x}_{0} \in \Gamma \\
v_{k}^{i-}\left(\mathbf{x}_{0}\right) & =-\frac{1}{2} \phi_{k}\left(\mathbf{x}_{0}\right)+\frac{1}{8 \pi} \int_{\Gamma} K_{j k}^{S}\left(\mathbf{y}, \mathbf{x}_{0}\right) \phi_{j}(\mathbf{y}) d \Gamma(\mathbf{y}) \\
& +\frac{1}{8 \pi} \int_{\Gamma} \mathcal{G}_{k j}\left(\mathbf{x}_{0}-\mathbf{y}\right) h_{j}(\mathbf{y}) d \Gamma(\mathbf{y}), \quad \mathbf{x}_{0} \in \Gamma
\end{aligned}
$$

By imposing the boundary condition (2.10) to the fields $\mathbf{v}^{e}$ and $\mathbf{v}^{i}$ given by the boundary integral representations (5.1) and (5.3), we obtain the Fredholm integral equations of the second kind:

$$
\begin{aligned}
\phi_{k}(\mathbf{x}) & +\frac{1}{8 \pi} \int_{\Gamma} K_{j k}^{c}\left(\mathbf{y}, \mathbf{x}_{0}\right) \phi_{j}(\mathbf{y}) d \Gamma(\mathbf{y}) \\
& +\frac{1}{8 \pi} \int_{\Gamma} \mathcal{G}_{k j}^{c}\left(\mathbf{x}_{0}-\mathbf{y}\right) h_{j}(\mathbf{y}) d \Gamma(\mathbf{y})=-U_{k}^{\infty}, \quad \mathbf{x}_{0} \in \Gamma,
\end{aligned}
$$

where

$$
\begin{aligned}
& K_{j k}^{c}\left(\mathbf{y}, \mathbf{x}_{0}\right)=K_{j k}^{\chi^{2}}\left(\mathbf{y}, \mathbf{x}_{0}\right)-K_{j k}^{S}\left(\mathbf{y}, \mathbf{x}_{0}\right), \\
& \mathcal{G}_{j k}^{c}\left(\mathbf{x}_{0}-\mathbf{y}\right)=\mathcal{G}_{j k}^{\chi^{2}}\left(\mathbf{x}_{0}-\mathbf{y}\right)-\mathcal{G}_{j k}\left(\mathbf{x}_{0}-\mathbf{y}\right) .
\end{aligned}
$$

Now, using the continuity property of the normal stresses due to the double-layer potentials $\mathbf{W}(\cdot, \Phi)$ and $\mathbf{W}_{\chi^{2}, 3}(\cdot, \Phi)$ across the boundary $\Gamma$ (see the properties (4.39) and (4.58)), the jump formulas of the boundary traction due to a single-layer potential (i.e., the properties $(4.34),(4.35)$ and $(4.52))$, as well as the boundary integral representations (5.1)-(5.4), we deduce that

$$
\begin{aligned}
t_{k}^{e+}\left(\mathbf{x}_{0}\right)= & t_{k}^{\infty}\left(\mathbf{x}_{0}\right)-\frac{1}{2} h_{k}\left(\mathbf{x}_{0}\right)+\frac{1}{8 \pi} \int_{\Gamma} K_{k j}^{\chi^{2}}\left(\mathbf{x}_{0}, \mathbf{y}\right) h_{j}(\mathbf{y}) d \Gamma(\mathbf{y}) \\
+ & T_{k j}\left(\mathbf{W}_{\chi^{2}, 3}(\Phi)\right)\left(\mathbf{x}_{0}\right) n_{k}\left(\mathbf{x}_{0}\right), \quad \mathbf{x}_{0} \in \Gamma \\
t_{k}^{i-}\left(\mathbf{x}_{0}\right) & =\frac{1}{2} h_{k}\left(\mathbf{x}_{0}\right)+\frac{1}{8 \pi} \int_{\Gamma} K_{k j}^{S}\left(\mathbf{x}_{0}, \mathbf{y}\right) h_{j}(\mathbf{y}) d \Gamma(\mathbf{y}) \\
& +T_{k j}(\mathbf{W}(\Phi))\left(\mathbf{x}_{0}\right) n_{k}\left(\mathbf{x}_{0}\right), \quad \mathbf{x}_{0} \in \Gamma
\end{aligned}
$$

where $\mathbf{t}^{\infty}$ is the boundary traction due to the fields $\mathbf{U}^{\infty}$ and $p^{\infty}$, i.e., $\mathbf{t}^{\infty}=-p^{\infty} \mathbf{n}$ on $\Gamma$. Also, we have

$$
t_{k}^{e+}\left(\mathbf{x}_{0}\right)=\lim _{\substack{\mathbf{x} \rightarrow \mathbf{x}_{0} \in \Gamma \\ \mathbf{x} \in D_{e}}} T_{k j}\left(\mathbf{v}^{e}\right)(\mathbf{x}) n_{k}\left(\mathbf{x}_{0}\right), \quad t_{k}^{i-}\left(\mathbf{x}_{0}\right)=\lim _{\substack{\mathbf{x} \rightarrow \mathbf{x}_{0} \in \Gamma \\ \mathbf{x} \in D_{0}}} T_{k j}\left(\mathbf{v}^{i}\right)(\mathbf{x}) n_{k}\left(\mathbf{x}_{0}\right),
$$


where $\mathbf{T}\left(\mathbf{v}^{e}\right)$ and $\mathbf{T}\left(\mathbf{v}^{i}\right)$ are the stress tensors due to the external fields $\left(\mathbf{v}^{e}, p^{e}\right)$ and to the internal fields $\left(\mathbf{v}^{i}, p^{i}\right)$, respectively. In addition, $\mathbf{T}\left(\mathbf{W}_{\chi^{2}, 3}(\Phi)\right)$ and $\mathbf{T}(\mathbf{W}(\Phi))$ are the stress tensors corresponding to the double-layer potentials $\mathbf{W}_{\chi^{2}, 3}(\cdot, \Phi)$ and $\mathbf{W}(\cdot, \Phi)$.

Furthermore, making use of the boundary condition (2.11) and the relations (5.9) and (5.10), we get the equations

$$
\begin{aligned}
-h_{k}\left(\mathbf{x}_{0}\right) & +\frac{1}{8 \pi} \int_{\Gamma} K_{k j}^{c}\left(\mathbf{x}_{0}, \mathbf{y}\right) h_{i}(\mathbf{y}) d \Gamma(\mathbf{y}) \\
& +T_{k j}\left(\mathbf{W}^{c}(\Phi)\right)\left(\mathbf{x}_{0}\right) n_{j}\left(\mathbf{x}_{0}\right)=-t_{k}^{\infty}\left(\mathbf{x}_{0}\right), \quad \mathbf{x}_{0} \in \Gamma,
\end{aligned}
$$

where

$$
\mathbf{W}^{c}(\Phi)=\mathbf{W}_{\chi^{2}, 3}(\cdot, \Phi)-\mathbf{W}(\cdot, \Phi) .
$$

Note that the pressure field of the undisturbed flow $p^{\infty}$ has the form $p^{\infty}(\mathbf{x})=-\chi^{2} \mathbf{U}^{\infty} \cdot \mathbf{x}$, $\mathbf{x} \in D_{e}$, as follows from the Brinkman equation. In addition, the assumption $\Gamma \in C^{2, \alpha}$ yields that $\mathbf{t}^{\infty} \in C^{1, \beta}(\Gamma)$ for any $\beta \in(0, \alpha]$.

According to the above arguments, we have reduced the boundary value problem (2.6)(2.12) to the system of Fredholm integral equations of the second kind (5.7) and (5.11). In order to prove the existence and uniqueness result of the solution to this system in the space $C^{1, \lambda}(\Gamma) \times C^{\lambda}(\Gamma), \lambda \in(0, \alpha)$, we will show that the corresponding homogeneous system has only the trivial solution in the same space. Then the desired existence and uniqueness result follows directly from Fredholm's alternative (see e.g. [16]).

Let us now consider the homogeneous system of equations

$$
\begin{aligned}
\phi_{k}^{0}\left(\mathbf{x}_{0}\right) & +\frac{1}{8 \pi} \int_{\Gamma} K_{j k}^{c}\left(\mathbf{y}, \mathbf{x}_{0}\right) \phi_{j}^{0}(\mathbf{y}) d \Gamma(\mathbf{y}) \\
+ & \frac{1}{8 \pi} \int_{\Gamma} \mathcal{G}_{k j}^{c}\left(\mathbf{x}_{0}-\mathbf{y}\right) h_{j}^{0}(\mathbf{y}) d \Gamma(\mathbf{y})=0, \quad \mathbf{x}_{0} \in \Gamma \\
-h_{k}^{0}\left(\mathbf{x}_{0}\right) & +\frac{1}{8 \pi} \int_{\Gamma} K_{k j}^{c}\left(\mathbf{x}_{0}, \mathbf{y}\right) h_{j}^{0}(\mathbf{y}) d \Gamma(\mathbf{y}) \\
& +T_{k j}\left(\mathbf{W}^{c}\left(\Phi^{0}\right)\right)\left(\mathbf{x}_{0}\right) n_{j}\left(\mathbf{x}_{0}\right)=0, \quad \mathbf{x}_{0} \in \Gamma
\end{aligned}
$$

$k=1,2,3$.

Also let $\left(\Phi^{0}, \mathbf{h}^{0}\right) \in C^{1, \lambda}(\Gamma) \times C^{\lambda}(\Gamma)$ be an arbitrary solution to this system, and let $\left(\mathbf{u}^{e}, q^{e}\right)$ and $\left(\mathbf{u}^{i}, q^{i}\right)$ be the fields given by

$$
\begin{aligned}
u_{k}^{e}(\mathbf{x}) & =\frac{1}{8 \pi} \int_{\Gamma} K_{j k}^{\chi^{2}}(\mathbf{y}, \mathbf{x}) \phi_{j}^{0}(\mathbf{y}) d \Gamma(\mathbf{y}) \\
& +\frac{1}{8 \pi} \int_{\Gamma} \mathcal{G}_{k j}^{\chi^{2}}(\mathbf{x}-\mathbf{y}) h_{j}^{0}(\mathbf{y}) d \Gamma(\mathbf{y}), \quad \mathbf{x} \in \mathbb{R}^{3} \backslash \Gamma, \\
q^{e}(\mathbf{x}) & =\frac{1}{8 \pi} \int_{\Gamma} \Lambda_{j k}^{\chi^{2}}(\mathbf{x}-\mathbf{y}) \phi_{j}^{0}(\mathbf{y}) d \Gamma(\mathbf{y}) \\
& +\frac{1}{8 \pi} \int_{\Gamma} \Pi_{j}^{\chi^{2}}(\mathbf{x}-\mathbf{y}) h_{j}^{0}(\mathbf{y}) d \Gamma(\mathbf{y}), \quad \mathbf{x} \in \mathbb{R}^{3} \backslash \Gamma,
\end{aligned}
$$


and

$$
\begin{aligned}
u_{k}^{i}(\mathbf{x}) & =\frac{1}{8 \pi} \int_{\Gamma} K_{j k}^{S}(\mathbf{y}, \mathbf{x}) \phi_{j}^{0}(\mathbf{y}) d \Gamma(\mathbf{y}) \\
& +\frac{1}{8 \pi} \int_{\Gamma} \mathcal{G}_{k j}(\mathbf{x}-\mathbf{y}) h_{j}^{0}(\mathbf{y}) d \Gamma(\mathbf{y}), \quad \mathbf{x} \in \mathbb{R}^{3} \backslash \Gamma, \\
q^{i}(\mathbf{x}) & =\frac{1}{8 \pi} \int_{\Gamma} \Lambda_{j k}^{S}(\mathbf{x}-\mathbf{y}) \phi_{j}^{0}(\mathbf{y}) d \Gamma(\mathbf{y}) \\
& +\frac{1}{8 \pi} \int_{\Gamma} \Pi_{j}^{S}(\mathbf{x}-\mathbf{y}) h_{j}^{0}(\mathbf{y}) d \Gamma(\mathbf{y}), \quad \mathbf{x} \in \mathbb{R}^{3} \backslash \Gamma .
\end{aligned}
$$

In view of the properties $(4.24),(4.25),(4.44)$ and (4.45) it follows that these fields satisfy the equations

$$
\begin{aligned}
& \nabla \cdot \mathbf{u}^{e}=0, \quad-\nabla q^{e}+\left(\nabla^{2}-\chi^{2}\right) \mathbf{u}^{e}=\mathbf{0} \text { in } \mathbb{R}^{3} \backslash \Gamma, \\
& \nabla \cdot \mathbf{u}^{i}=0, \quad-\nabla q^{i}+\nabla^{2} \mathbf{u}^{i}=\mathbf{0} \text { in } \mathbb{R}^{3} \backslash \Gamma .
\end{aligned}
$$

In addition, from the properties (4.54) and (4.55) we find that

$$
\left(\left|\mathbf{u}^{e}\right|\left|\nabla \mathbf{u}^{e}\right|\right)(\mathbf{x})=o\left(|\mathbf{x}|^{-2}\right), \quad\left(\left|\mathbf{u}^{e}\right|\left|p^{e}\right|\right)(\mathbf{x})=o\left(|\mathbf{x}|^{-2}\right) \text { as }|\mathbf{x}| \rightarrow \infty .
$$

Therefore, the fields $\mathbf{u}^{e}$ and $q^{e}$ satisfy the identity (see e.g. [15], p. 24)

$$
\int_{D_{e}}\left(2 E_{j k}\left(\mathbf{u}^{e}\right) E_{j k}\left(\mathbf{u}^{e}\right)+\chi^{2}\left|\mathbf{u}^{e}\right|^{2}\right) d \mathbf{x}=-\int_{\Gamma} u_{k}^{e+}(\mathbf{x}) t_{k}^{+}\left(\mathbf{u}^{e}\right)(\mathbf{x}) d \Gamma(\mathbf{x}),
$$

where $t_{k}^{ \pm}\left(\mathbf{u}^{e}\right)=T_{k j}^{ \pm}\left(\mathbf{u}^{e}\right) n_{k}$, and

$$
T_{k j}\left(\mathbf{u}^{e}\right)=-q^{e} \delta_{k j}+2 E_{k j}\left(\mathbf{u}^{e}\right), E_{j k}\left(\mathbf{u}^{e}\right)=\frac{1}{2}\left(\frac{\partial u_{j}^{e}}{\partial x_{k}}+\frac{\partial u_{k}^{e}}{\partial x_{j}}\right), \quad j, k=1,2,3 .
$$

Similarly, the fields $\mathbf{u}^{i}$ and $q^{i}$ satisfy the identity (see e.g. [15], p. 15)

$$
2 \int_{D_{0}} E_{j k}\left(\mathbf{u}^{i}\right) E_{j k}\left(\mathbf{u}^{i}\right) d \mathbf{x}=\int_{\Gamma} u_{k}^{i-}(\mathbf{x}) t_{k}^{-}\left(\mathbf{u}^{i}\right)(\mathbf{x}) d \Gamma(\mathbf{x}),
$$

where $t_{k}^{ \pm}\left(\mathbf{u}^{i}\right)=T_{k j}^{ \pm}\left(\mathbf{u}^{i}\right) n_{k}$, and

$$
T_{k j}\left(\mathbf{u}^{i}\right)=-q^{i} \delta_{k j}+2 E_{k j}\left(\mathbf{u}^{i}\right), E_{j k}\left(\mathbf{u}^{i}\right)=\frac{1}{2}\left(\frac{\partial u_{j}^{i}}{\partial x_{k}}+\frac{\partial u_{k}^{i}}{\partial x_{j}}\right), \quad j, k=1,2,3 .
$$

Now, taking into account the fact that $\left(\Phi^{0}, \mathbf{h}^{0}\right)$ is a solution to the system of equations (5.13) and (5.14), and making use of the relations (4.31)-(4.35) and (4.50)-(4.52), we obtain the properties

$$
\begin{aligned}
u_{k}^{e+} & =u_{k}^{i-} \text { on } \Gamma, \\
t_{k}^{+}\left(\mathbf{u}^{e}\right) & =t_{k}^{-}\left(\mathbf{u}^{i}\right) \text { on } \Gamma,
\end{aligned}
$$

in view of which we get the equality

$$
\int_{\Gamma} u_{k}^{e+}(\mathbf{x}) t_{k}^{+}\left(\mathbf{u}^{e}\right)(\mathbf{x}) d \Gamma=\int_{\Gamma} u_{k}^{i-}(\mathbf{x}) t_{k}^{-}\left(\mathbf{u}^{i}\right)(\mathbf{x}) d \Gamma .
$$


The properties (5.22), (5.23) and (5.26) yield that

$$
\int_{D_{e}}\left(2 E_{j k}\left(\mathbf{u}^{e}\right) E_{j k}\left(\mathbf{u}^{e}\right)+\chi^{2}\left|\mathbf{u}^{e}\right|^{2}\right) d \mathbf{x}=-2 \int_{D_{0}} E_{j k}\left(\mathbf{u}^{i}\right) E_{j k}\left(\mathbf{u}^{i}\right) d \mathbf{x} .
$$

Consequently, we have

$$
\begin{gathered}
\mathbf{u}^{e}=\mathbf{0} \text { in } D_{e} \\
\frac{\partial u_{j}^{i}}{\partial x_{k}}+\frac{\partial u_{k}^{i}}{\partial x_{j}}=0 \text { in } D_{0}, \quad j, k=1,2,3 .
\end{gathered}
$$

The general solution of the system of equations (5.29) represents a rigid body motion field, i.e., it has the form (see e.g. [15], p. 17)

$$
\mathbf{u}^{i}(\mathbf{x})=\mathbf{A}+\mathbf{B} \times \mathbf{x}, \quad \mathbf{x} \in D_{0}
$$

where $\mathbf{A}$ and $\mathbf{B}$ are constant vectors (or functions which depend only on $t$ ).

In addition, in view of the second of equations (5.19) and from the fact that the pressure field $p^{e}$ vanishes at infinity, we deduce that

$$
p^{e}=0 \text { in } D_{e} .
$$

From the properties (5.24) and (5.28) it follows that

$$
u_{k}^{i-}=u_{k}^{e+}=0 \text { on } \Gamma \text {. }
$$

This result together with the property (5.30) yield that $\mathbf{A}=\mathbf{B}=\mathbf{0}$, i.e.,

$$
\mathbf{u}^{i}=\mathbf{0}, \quad q^{i}=c \text { in } D_{0},
$$

where $c \in \mathbb{R}$.

On the other hand, from the relations (5.25), (5.28) and (5.31) we get

$$
t_{k}^{-}\left(\mathbf{u}^{i}\right)=t_{k}^{+}\left(\mathbf{u}^{e}\right)=0 \text { on } \Gamma
$$

and hence the constant $c$ must be equal to zero, i.e.,

$$
\mathbf{u}^{i}=0, \quad q^{i}=0 \text { in } D_{0} .
$$

Now, using the jump formula

$$
\mathbf{u}^{e+}-\mathbf{u}^{e-}=\Phi^{0} \text { on } \Gamma
$$

(see the properties (4.50) and (4.51)) as well as the result (5.28), we deduce that

$$
\mathbf{u}^{e-}=-\Phi^{0} \text { on } \Gamma \text {. }
$$

Similarly, from the jump formula

$$
\mathbf{u}^{i+}-\mathbf{u}^{i-}=\Phi^{0} \text { on } \Gamma
$$

(see the properties (4.31)-(4.33)) as well as the result (5.32), we find that

$$
\mathbf{u}^{i+}=\Phi^{0} \text { on } \Gamma \text {. }
$$

On the other hand, from the relations (4.52) we deduce that the boundary traction due to the fields $\mathbf{u}^{e}$ and $q^{e}$ has a jump across $\Gamma$ given by the formula

$$
\mathbf{t}^{+}\left(\mathbf{u}^{e}\right)-\mathbf{t}^{-}\left(\mathbf{u}^{e}\right)=-\mathbf{h}^{0} \text { on } \Gamma \text {. }
$$


But $\mathbf{t}^{+}\left(\mathbf{u}^{e}\right)=\mathbf{0}$ on $\Gamma$ and hence

$$
\mathbf{t}^{-}\left(\mathbf{u}^{e}\right)=\mathbf{h}^{0} \text { on } \Gamma \text {. }
$$

With similar kinds of arguments as before, we get the relation

$$
\mathbf{t}^{+}\left(\mathbf{u}^{i}\right)=-\mathbf{h}^{0} \text { on } \Gamma .
$$

In addition, the fields $\left(\mathbf{u}^{e}, q^{e}\right)$ satisfy the identity

$$
\int_{D_{0}}\left(2 E_{j k}\left(\mathbf{u}^{e}\right) E_{j k}\left(\mathbf{u}^{e}\right)+\chi^{2}\left|\mathbf{u}^{e}\right|^{2}\right) d \mathbf{x}=\int_{\Gamma} u_{k}^{e-}(\mathbf{x}) t_{k}^{-}\left(\mathbf{u}^{e}\right)(\mathbf{x}) d \Gamma(\mathbf{x})
$$

and, in view of the properties (5.36) and (5.39), this identity takes the form

$$
\int_{D_{0}}\left(2 E_{j k}\left(\mathbf{u}^{e}\right) E_{j k}\left(\mathbf{u}^{e}\right)+\chi^{2}\left|\mathbf{u}^{e}\right|^{2}\right) d \mathbf{x}=-\int_{\Gamma} \Phi^{0} \cdot \mathbf{h}^{0} d \Gamma .
$$

Similarly, the fields $\left(\mathbf{u}^{i}, q^{i}\right)$ satisfy the identity

$$
2 \int_{D_{e}} E_{j k}\left(\mathbf{u}^{i}\right) E_{j k}\left(\mathbf{u}^{i}\right) d \mathbf{x}=-\int_{\Gamma} u_{k}^{i+}(\mathbf{x}) t_{k}^{+}\left(\mathbf{u}^{i}\right)(\mathbf{x}) d \Gamma(\mathbf{x}),
$$

which, in view of the properties (5.37) and (5.40), becomes

$$
2 \int_{D_{e}} E_{j k}\left(\mathbf{u}^{i}\right) E_{j k}\left(\mathbf{u}^{i}\right) d \mathbf{x}=\int_{\Gamma} \Phi^{0} \cdot \mathbf{h}^{0} d \Gamma .
$$

Therefore, from the identities (5.42) and (5.44) we obtain the following equality:

$$
\int_{D_{0}}\left(2 E_{j k}\left(\mathbf{u}^{e}\right) E_{j k}\left(\mathbf{u}^{e}\right)+\chi^{2}\left|\mathbf{u}^{e}\right|^{2}\right) d \mathbf{x}=-2 \int_{D_{e}} E_{j k}\left(\mathbf{u}^{i}\right) E_{j k}\left(\mathbf{u}^{i}\right) d \mathbf{x}
$$

which yields that

$$
\mathbf{u}^{e}=\mathbf{0} \text { in } D_{0}
$$

and

$$
\mathbf{u}^{i}=\mathbf{0} \text { in } D_{e} .
$$

The property (5.47), the Stokes equation $-\nabla q^{i}+\nabla^{2} \mathbf{u}^{i}=\mathbf{0}$ in $D_{e}$, and the fact that the pressure field $q^{i}$ vanishes at infinity yield the additional result

$$
q^{i}=0 \text { in } D_{e} .
$$

Now, taking into account the relations (5.36) and (5.46) we deduce that

$$
\Phi^{0}=\mathbf{0} \text { on } \Gamma .
$$

In addition, according to the relations (5.40), (5.47) and (5.48) we find that

$$
\mathbf{h}^{0}=\mathbf{0} \text { on } \Gamma \text {. }
$$

Consequently, from the properties (5.49) and (5.50) we deduce that the homogeneous system of equations (5.13) and (5.14) has only the trivial solution in the space $C^{1, \lambda}(\Gamma) \times$ $C^{\lambda}(\Gamma)$ and hence, in view of Fredholm's alternative, the system of Fredholm integral equations of the second kind (5.7) and (5.11) has a unique solution $(\Phi, \mathbf{h}) \in C^{1, \lambda}(\Gamma) \times$ $C^{\lambda}(\Gamma)$.

The previous arguments lead to the following existence and uniqueness result: 
Theorem 5.1. Let $\Gamma$ be a close Lyapunov surface of class $C^{2, \alpha}$ in $\mathbb{R}^{3}, \alpha \in(0,1]$, and let $\lambda \in(0, \alpha)$. Then the system of Fredholm integral equations of the second kind (5.7) and (5.11) has a unique solution $(\Phi, \mathbf{h}) \in C^{1, \lambda}(\Gamma) \times C^{\lambda}(\Gamma)$, and the boundary integral representations (5.1)-(5.4), obtained with the vector densities $\Phi$ and $\mathbf{h}$, determine the unique classical solution

$$
\left(\left(\mathbf{v}^{e}, p^{e}\right),\left(\mathbf{v}^{i}, p^{i}\right)\right) \in\left(\left(C^{2}\left(D_{e}\right) \cap C^{1}\left(\bar{D}_{e}\right)\right) \times C^{1}\left(\bar{D}_{e}\right)\right) \times\left(\left(C^{2}\left(D_{0}\right) \cap C^{1}\left(\bar{D}_{0}\right)\right) \times C^{1}\left(\bar{D}_{0}\right)\right)
$$

to the boundary value problem (2.6)-(2.12).

6. Conclusion. In this paper we have developed an indirect boundary integral formulation in order to show the existence and uniqueness result of the classical solution to a boundary value problem which describes the three-dimensional viscous flow in a granular material with a void. We have assumed that the flow in the granular material is governed by the continuity and Brinkman equations, and that the flow inside the void is described by the continuity and Stokes equations. The desired existence and uniqueness result is obtained by using the potential theory for both Stokes and Brinkman equations, as well as certain results of the theory of integral equations. The corresponding numerical solution can be obtained by applying a Boundary Element Method (BEM) to the system of boundary integral equations. Because of the wide range of applications of BEM to flow in porous media, the boundary integral formulation of the present problem is very useful. In particular, an extensive application of BEM to groundwater flow is given by Liggett and Liu [19], Cheng and Ouazar [4]. Since the power of BEM allows one to deal with any arbitrary geometry, the authors propose to get the corresponding numerical results for the presence void of arbitrary shape in future investigations.

\section{REFERENCES}

[1] G. S. Beavers, D.D. Joseph, Boundary conditions at a naturally permeable wall, J. Fluid Mech., 30 (1967), 197-207.

[2] V. Botte, H. Power, A second kind integral equation formulation for three-dimensional interior flows at low Reynolds number, Bound. Elem. Commun., 6 (1995), 163-166.

[3] F. J. Briceno, H. Power, The completed integral equation approach for the numerical solution of the motion of $N$ solid particles in the interior of a deformable viscous drop, Eng. Anal. Bound. Elem., 28 (2004), 367-413.

[4] A. D. H. Cheng, D. Ouazar, Ground Water Flow, Elsevier, 1993.

[5] W. D. Collins, Note on a sphere theorem for the axisymmetric Stokes flow of a viscous fluid, Mathematika, 5 (1958), 118-121. MR0104424 (21:3179)

[6] L. C. Evans, Partial Differential Equations, American Mathematical Society, vol. 19, Providence, 2002. MR1625845 (99e:35001)

[7] T. M. Fischer, G. C. Hsiao, W. L. Wendland, On two-dimensional slow viscous flows past obstacles in a half-plane, Proc. Royal Society of Edinburgh, 104A (1986) 205-215. MR877902 (88c:76022)

[8] J. J. L. Higdon, M. Kojima, On the calculation of Stokes flow past porous particles, Multiphase Flow, 7 (1981), 719-727.

[9] I. P. Jones, Low Reynolds number flow past spherical shell, Proc. Camb. Phil. Soc., 73 (1973), $231-238$.

[10] S. J. Karrila, S. Kim, Integral equations of the second kind for Stokes flow: direct solution for physical variables and removal of inherent accuracy limitations, Chem. Engng. Commun., 82 (1989), 123-161.

[11] M. Kohr, An indirect boundary integral method for a Stokes flow problem, Comput. Methods Appl. Mech. Engrg., 190 (2000), 487-497. MR1800567 (2001j:76077) 
[12] M. Kohr, An indirect boundary integral method for an oscillatory Stokes flow problem, Int. J. Math. Math. Sci., 47 (2003), 2961-2976. MR2010743 (2004h:76059)

[13] M. Kohr, A mixed boundary value problem for the unsteady Stokes system in a bounded domain in $\mathbb{R}^{n}$, Engineering Analysis with Boundary Elements, 29 (2005), 936-943.

[14] M. Kohr, The Dirichlet problems for the unsteady Stokes system in bounded and exterior domains in $\mathbb{R}^{n}$, Mathematische Nachrichten, 280 (2007), 534-559.

[15] M. Kohr, I. Pop, Viscous Incompressible Flow for Low Reynolds Numbers, WIT Press, Southampton, UK, 2004. MR2091145 (2005k:76032)

[16] R. Kress, Linear Integral Equations, Springer, Berlin, 1989. MR1007594 (90j:45001)

[17] V. D. Kupradze, T. G. Gegelia, M. O. Basheleishvili, T. V. Burchuladze, Three-Dimensional Problems of the Mathematical Theory of Elasticity and Thermoelasticity, North Holland, Amsterdam, 1979. MR530377 (80h:73002)

[18] O. A. Ladyzhenskaya, The Mathematical Theory of Viscous Incompressible Flow, Gordon and Breach Science Publishers, New York, 1969. MR0254401 (40:7610)

[19] J. A. Liggett, P. L. F. Liu, The Boundary Integral Equation Method for Porous Media Flow, George Allen and Unwinn, London 1983. MR732790 (84m:76086)

[20] P. Maremonti, R. Russo, G. Starita, On the Stokes equations: the boundary value problem. In: Advances in Fluid Dynamics, Quad. Mat. (Aracne, Rome), 1999, 69-140. MR1770189 (2001g:35212)

[21] G. Neale, N. Epstein, W. Nader, Creeping flow relative to permeable spheres, Chem. Eng. Sci., 28 (1973), 1865-1874. (Erratum: 1974, 29, 1352).

[22] J. A. Ochoa-Tapia, S. Whitaker, Momentum transfer at the boundary between a porous medium and a homogeneous fluid - I, Theoretical development, Int. J. Heat and Mass Transfer, 38 (1995), $2635-2646$.

[23] J. A. Ochoa-Tapia, S. Whitaker, Momentum transfer at the boundary between a porous medium and a homogeneous fluid - II, Comparison with experiment, Int. J. Heat and Mass Transfer, 38 (1995), 2647-2655.

[24] Y. U. Qin, P. N. Kaloni, Creeping flow past a porous spherical shell, Z. Angew. Math. Mech., 73 (1993), 77-84. MR1211619 (93m:76024)

[25] B. S. Padmavathi, T. Amaranath, S. D. Nigam, Stokes flow past a porous sphere using Brinkman's model, Z. Angew. Math. Phys., 44 (1993), 929-939. MR1241641 (94h:76022)

[26] D. Palanippan, Arbitrary Stokes flow past a porous sphere, Mech. Res. Comm., 20 (1993), 309-317.

[27] H. Power, The completed double layer boundary integral equation method for two-dimensional Stokes flow, IMA J. Appl. Math., 51 (1993), 123-145. MR1244192 (94i:76020)

[28] H. Power, G. Miranda, Second kind integral equation formulation of Stokes flows past a particle of arbitrary shape, SIAM J. Appl. Math., 47 (1987), 689-698. MR898827 (88h:76021)

[29] H. Power, L. C. Wrobel, Boundary Integral Methods in Fluid Mechanics, WIT Press, Computational Mechanics Publications, Southampton, 1995.

[30] O. Sano, Viscous flow past a cylidrical hole bored inside porous media - with application to measurement of the velocity of subterranean water by the single boring method, Nagare, 2 (1983), $252-259$.

[31] G. P. Raja Sekhar, B. S. Padmavathi, T. Amaranath, Complete general solution of the Brinkman Equations, Z. Angew. Math. Mech., 77 (1997), 555-556. MR1466445

[32] G. P. Raja Sekhar, T. Amaranath, A Stokes flow inside a porous spherical shell, Z. Angew. Math. Phys., 51 (2000), 481-490. MR1762703 (2001a:76047)

[33] G. P. Raja Sekhar, O. Sano, Viscous flow past a circular/spherical void in porous medium-an application to measurement of the velocity of ground water by single boring method, J. Phys. Soc. Jpn., 69 (2000), 37-42.

[34] G. P. Raja Sekhar, O. Sano, Two-dimensional viscous flow past a slightly deformed circular cavity in a porous medium, Fluid. Dyn. Res., 28 (2001), 281-293.

[35] G. P. Raja Sekhar, O. Sano, Two-dimensional viscous flow in a granular material with a void of arbitrary shape, Physics of Fluids, 15 (2003), 554-567.

[36] G. P. Raja Sekhar, M. K. Partha, P. V. S. N. Murthy, Viscous flow past a spherical void in porous media - Effect of stress jump boundary condition, J. Porous Media, 9 (2006), 745-767.

[37] K. B. Ranger, The Stokes drag for asymmetric flow past a spherical cap, Z. Angew. Math. Phys., 24 (1973), 801-809.

[38] R. Shail, A note on some axisymmetric Stokes flow within a sphere, QJMAM, 40 (1987), 223-233. MR894200 (88c:76025) 
[39] W. Varnhorn, An explicit potential theory for the Stokes resolvent boundary value problems in three dimensions, Manuscripta Math., 70 (1991), 339-361. MR1092141 (91k:31011)

[40] W. Varnhorn, The Stokes Equations, Akademie Verlag, Berlin, 1994. MR1282728 (95e:35162)

[41] W. Varnhorn, The boundary value problems of the Stokes resolvent equations in $n$ dimensions, Math. Nachr., 269-270 (2004), 210-230. MR2074782 (2005e:35192)

[42] J. Wloka, Funktionalanalysis und Anwendungen, de Gruyter, 1971. MR0467224 (57:7088)

[43] G. K. Youngren, A. Acrivos, Stokes flow past a particle of arbitrary shape: a numerical method of solution, J. Fluid Mech., 69 (1975), 377-403. MR0398289 (53:2142) 